\title{
Search for large extra dimensions in the diphoton final state at the Large Hadron Collider
}

\section{CMS collaboration}

E-mail: cms-publication-committee-chair@cern.ch

ABSTRACT: A search for large extra spatial dimensions via virtual-graviton exchange in the diphoton channel has been carried out with the CMS detector at the LHC. No excess of events above the standard model expectations is found using a data sample collected in proton-proton collisions at $\sqrt{s}=7 \mathrm{TeV}$ and corresponding to an integrated luminosity of $36 \mathrm{pb}^{-1}$. New lower limits on the effective Planck scale in the range of $1.6-2.3 \mathrm{TeV}$ at the $95 \%$ confidence level are set, providing the most restrictive bounds to date on models with more than two large extra dimensions.

KEYwORDS: Hadron-Hadron Scattering 


\section{Contents}

1 Introduction 1

2 The CMS detector 2

3 Event reconstruction and selection 3

4 Signal and background estimation 4

$\begin{array}{lll}5 & \text { Results } & 7\end{array}$

6 Conclusions $\quad 9$

\section{Introduction}

Compact large extra dimensions (ED) are an intriguing proposed solution to the hierarchy problem of the standard model (SM), which refers to the puzzling fact that the fundamental scale of gravity $M_{\mathrm{Pl}} \sim 10^{19} \mathrm{GeV}$ is so much higher than the electroweak symmetry breaking scale $\sim 10^{3} \mathrm{GeV}$. With such a difference in scales, it is difficult to protect the Higgs boson mass from radiative corrections without a very high degree of fine-tuning. The original proposal to use ED to solve the hierarchy problem was presented by ArkaniHamed, Dimopoulos, and Dvali (ADD) [1,2]. They posited a scenario wherein the SM is constrained to the common $3+1$ space-time dimensions, while gravity is free to propagate through the entire multidimensional space. Thus, the gravitational flux in $3+1$ dimensions is effectively diluted by virtue of the multidimensional Gauss's Law. The fundamental Planck scale $M_{\mathrm{D}}$ is therefore related to the apparent scale $M_{\mathrm{Pl}}$ according to the formula $M_{\mathrm{D}}^{n_{\mathrm{ED}}+2}=M_{\mathrm{Pl}}^{2} / r^{n_{\mathrm{ED}}}$, where $r$ and $n_{\mathrm{ED}}$ are the size and number of the extra dimensions, respectively. A review of current limits on the ADD model can be found in ref. [3].

Phenomenologically, this large ED scenario results in $s$-channel production of massive, virtual Kaluza-Klein (KK) graviton states, which can decay into two photons [4]. Decays to fermions are suppressed relative to photons because the graviton is spin- 2 and the fermions cannot be produced in the $s$ wave. Because the KK gravitons propagate through the compact extra dimensions, their wavefunction must satisfy periodic boundary conditions, which result in discrete energy levels with modal spacing of the order of the inverse ED size, from $1 \mathrm{meV}$ to $100 \mathrm{MeV}$. This results in an apparent continuum spectrum of diphotons, rather than distinct resonances.

Summing over KK modes results in a divergence in the cross section, so an ultraviolet (UV) cutoff scale $M_{\mathrm{S}}$ is needed. This scale is related to — but potentially different from the fundamental Planck scale $M_{\mathrm{D}}$, as the precise relationship depends on the UV completion of the effective theory. The effects of virtual-graviton production on the cross section are 
parameterized by the single variable $\eta_{G}=\mathcal{F} / M_{\mathrm{S}}^{4}$, where $\mathcal{F}$ is an order-unity dimensionless parameter, for which several conventional assumptions exist:

$$
\begin{aligned}
& \mathcal{F}=1 \quad \text { (Giudice, Rattazzi, and Wells, GRW [5]), } \\
& \mathcal{F}=\left\{\begin{array}{ll}
\log \left(\frac{M_{\mathrm{S}}^{2}}{\hat{s}}\right) \text { if } n_{\mathrm{ED}}=2 \\
\frac{2}{\left(n_{\mathrm{ED}}-2\right)} \text { if } n_{\mathrm{ED}}>2
\end{array} \quad\right. \text { (Han, Lykken, and Zhang, HLZ [6]), } \\
& \mathcal{F}= \pm \frac{2}{\pi} \quad(\text { Hewett }[7]),
\end{aligned}
$$

where $\sqrt{\hat{s}}$ is the center-of-mass energy of the hard parton-parton collision. We note that the HLZ convention contains an explicit dependence on the number of extra dimensions.

Searches for extra dimensions via virtual-graviton effects have been conducted at HERA, LEP, and the Tevatron (refs. [3, 8] contain recent reviews of these searches). The most stringent previously published limits on $M_{\mathrm{S}}$ come from the D0 measurements in the dijet [9] and diphoton plus dielectron [10] channels, which exclude values of $M_{\mathrm{S}}$ lower than $1.3-2.1 \mathrm{TeV}$ at $95 \%$ confidence level (CL), depending on $n_{\mathrm{ED}}$.

In this paper, we present a search for virtual-graviton contributions in the diphoton final state, using a data sample corresponding to an integrated luminosity of $36 \mathrm{pb}^{-1}$, collected in pp collisions at $\sqrt{s}=7 \mathrm{TeV}$ at the CERN Large Hadron Collider (LHC) with the Compact Muon Solenoid (CMS) detector.

\section{The CMS detector}

CMS is a general-purpose detector designed to study proton collisions at the LHC and is described in detail in ref. [11]. The detector consists of an all-silicon tracker, an electromagnetic calorimeter (ECAL), and a hadronic sampling calorimeter (HCAL), all contained inside a large-bore $3.8 \mathrm{~T}$ superconducting solenoid. In the central region, the tracker consists of three layers of silicon pixel detectors, followed by 10 layers of single- and double-sided silicon-strip detectors. The calorimeter towers are projective and finely segmented, with $\Delta \eta \approx \Delta \phi \approx 0.087$ in the central region. Moreover, each tower consists of a five-by-five transverse grid of ECAL crystals $(\Delta \eta \approx \Delta \phi \approx 0.0174)$, allowing precise reconstruction of the $\mathrm{e} / \gamma$ position and energy. Here, the pseudorapidity $\eta$ is defined as $-\ln \left(\tan \frac{\theta}{2}\right)$, where $\theta$ is the polar angle with respect to the direction of the counterclockwise beam, and $\phi$ is the azimuthal angle. Beyond the solenoid there are four layers of muon detectors, which are interspersed throughout the steel return yoke of the magnet. The instantaneous luminosity is measured with a relative uncertainty of $4 \%$ using information from forward hadronic calorimeters [12].

The CMS trigger system consists of two levels. The first level (L1), composed of custom hardware, uses information from the calorimeters and muon detectors to select the most interesting events for more refined selection and analysis at a rate of up to $80 \mathrm{kHz}$. The software-based High Level Trigger further decreases the rate to a maximum of $\sim 300 \mathrm{~Hz}$ for data storage. Events for the control samples used in this analysis were collected through a single-photon trigger, where the photon was required to have a transverse energy $E_{\mathrm{T}} \equiv$ 
$E \sin \theta$ of at least 20 or $30 \mathrm{GeV}$, depending on the data collection period. Events in the signal sample were also initially collected through a single $E_{\mathrm{T}}>20 \mathrm{GeV}$ photon trigger. However, as the instantaneous luminosity increased, we switched to a double-photon trigger requiring both photons to have $E_{\mathrm{T}}>22 \mathrm{GeV}$.

\section{Event reconstruction and selection}

We first require that an event be consistent with a pp collision and have at least one wellreconstructed primary vertex [13]. We then reconstruct photons with $E_{\mathrm{T}}>30 \mathrm{GeV}$ in the ECAL barrel fiducial region $(|\eta|<1.44)$ by clustering electromagnetic energy depositions in the ECAL. The ECAL clusters are five crystals wide in $\eta$ and a variable length in $\phi$ to capture associated electromagnetic energy from possible photon conversions in the tracker. If hits are present in the pixel detector consistent with an electron track whose momentum and location is similar to the energy and location of the ECAL cluster, then the cluster is rejected as a photon candidate. In 2010 collision data, the ECAL has an energy resolution better than $1 \%$ in the barrel for unconverted photons with $E_{\mathrm{T}}>20 \mathrm{GeV}$ [14].

Hadronic jets can be misidentified as photons when their leading hadron is a hard $\pi^{0}$ or $\eta$. We reduce the misidentification rate by placing the following restrictions on the isolation of the cluster: (i) the hadronic energy within $\Delta R<0.15$ of the cluster must be less than $5 \%$ of its electromagnetic energy, where $\Delta R \equiv \sqrt{\Delta \phi^{2}+\Delta \eta^{2}} \equiv \sqrt{\left(\phi-\phi_{\gamma}\right)^{2}+\left(\eta-\eta_{\gamma}\right)^{2}}$; (ii) the $\Sigma E_{\mathrm{T}}$ of HCAL energy surrounding the cluster within $0.15<\Delta R<0.40$ must be less than $2.2 \mathrm{GeV}+0.0025 E_{\mathrm{T}}$; (iii) the scalar sum of the transverse momentum of tracks, $\Sigma p_{\mathrm{T}}$, associated with the primary event vertex surrounding the cluster within a hollow cone of $0.04<\Delta R<0.40$ must be less than $2.0 \mathrm{GeV}+0.001 E_{\mathrm{T}}$, where $E_{\mathrm{T}}$ is the photon transverse energy (a rectangular strip of $\Delta \eta \times \Delta \phi=0.015 \times 0.400$ at the front face of the ECAL is excluded from the track $p_{\mathrm{T}}$ summation to allow for photons that convert into $\mathrm{e}^{+} \mathrm{e}^{-}$ pairs); and (iv) the $\Sigma E_{\mathrm{T}}$ of ECAL energy surrounding the cluster within $0.06<\Delta R<0.40$ (and excluding a strip of $\Delta \eta \times \Delta \phi=0.04 \times 0.400$ ) must be less than $4.2 \mathrm{GeV}+0.006 E_{\mathrm{T}}$. We also require that the shower shape in $\eta, \sigma_{\eta \eta}$, be consistent with a photon. The $\sigma_{\eta \eta}$ variable is a modified second moment of the electromagnetic energy cluster about its mean $\eta$ position, defined in ref. [15]. Topological and timing criteria suppress noise present in the ECAL $[14,16]$.

We reconstruct two photons using the selection described above and require that the invariant mass of the two photons satisfies $M_{\gamma \gamma}>60 \mathrm{GeV}$. The invariant mass and photon pseudorapidity selection criteria are optimized to produce the highest sensitivity for values of $M_{\mathrm{S}}$ and $n_{\mathrm{ED}}$ to which the present data is sensitive. The optimization is performed first with a fixed $M_{\gamma \gamma}$ requirement and a floating $|\eta|$ requirement. We then alternate, floating the $|\eta|$ requirement and fixing the $M_{\gamma \gamma}$ requirement. This iterative optimization is continued until we converge on a final choice of $|\eta|<1.44$ and $M_{\gamma \gamma}>500 \mathrm{GeV}$. The optimal choice for $|\eta|$ was very close to the ECAL barrel-endcap boundary, so we chose the edge of the transition region into the boundary for simplicity. This defines the signal region. The intervals $60<M_{\gamma \gamma}<200 \mathrm{GeV}$ and $200<M_{\gamma \gamma}<500 \mathrm{GeV}$ define the control and intermediate regions of the data, respectively. 
The photon reconstruction and identification efficiency is measured in Monte Carlo (MC) simulation and corrected using a data/MC scale factor of $1.010 \pm 0.012$ derived from studying $Z \rightarrow e^{+} e^{-}$events. The final efficiency is roughly constant as a function of the photon $E_{\mathrm{T}}$ and $\eta$. The efficiency for an $E_{\mathrm{T}}>30 \mathrm{GeV}$ photon with $|\eta|<1.44$ is $(87.8 \pm 2.3) \%$, where the dominant systematic uncertainty is chosen to cover the variation as a function of $E_{\mathrm{T}}$ and $\eta$. Therefore, the corresponding diphoton reconstruction and identification efficiency is $(77.1 \pm 4.0) \%$.

\section{Signal and background estimation}

We simulate ED in the ADD model using the SHERPA (v1.1.2) [17] MC generator, which samples different operating points in $M_{\mathrm{S}}$ and $n_{\mathrm{ED}}$, followed by a fast parametric simulation of the CMS detector [18]. A fast simulation is adequate for describing multiphoton final states and has been extensively validated using full simulation of the detector via GEANT4 [19]. The simulation includes both SM diphoton production and signal diphoton production via KK-graviton exchange, in order to account for the interference effects. We use CETQ6L1 [20] parton distribution functions (PDF) in the simulation. The leading order (LO) SHERPA cross sections are multiplied by a next-to-leading order (NLO) $K$ factor of $1.3 \pm 0.1[21,22]$, where the systematic uncertainty covers the variation of the $K$ factor with the diphoton mass. Additionally, a 1.5\% relative uncertainty on the signal acceptance is included to account for uncertainty due to the PDF.

Backgrounds due to the mimicking of a photon signal by a jet are small in the signal region. There are two sources of these backgrounds from isolated photon misidentification which we consider: multijet production and prompt photon production (i.e., photons from $\gamma+$ jets). In particular, we measure a misidentification rate, defined as the ratio of the number of isolated photons to non-isolated photons in a sample, where the non-isolated photons are selected similarly to the isolated photons except that they fail one of the isolation or shower-shape criteria. The samples corresponding to numerator and denominator are defined such that they are mutually exclusive. The misidentification rate is measured in an EM enriched sample (triggered on an electromagnetic cluster) and is then applied to the observed events with one or more non-isolated photons, resulting in a prediction of the dijet and $\gamma+$ jet backgrounds.

Because the control sample in which we measure the misidentification rate may contain some number of real, isolated photons that "contaminate" the misidentification-rate numerator, we correct for the numerator purity on a sample-by-sample basis. This is done by releasing the $\sigma_{\eta \eta}$ requirement and fitting the numerator sample for the fraction of prompt photons using one-dimensional probability density histograms ("templates") in $\sigma_{\eta \eta}$. The signal template is constructed from MC simulation, and the background template is constructed from reconstructed photons that fail one or more of the isolation criteria. The measured misidentification rate falls from $28 \%$ at $E_{\mathrm{T}}=30 \mathrm{GeV}$ to $2 \%$ at $E_{\mathrm{T}}=120 \mathrm{GeV}$. We use two other complementary techniques (using converted photons and an isolation template to estimate prompt-photon contamination) to bound the misidentification rate 

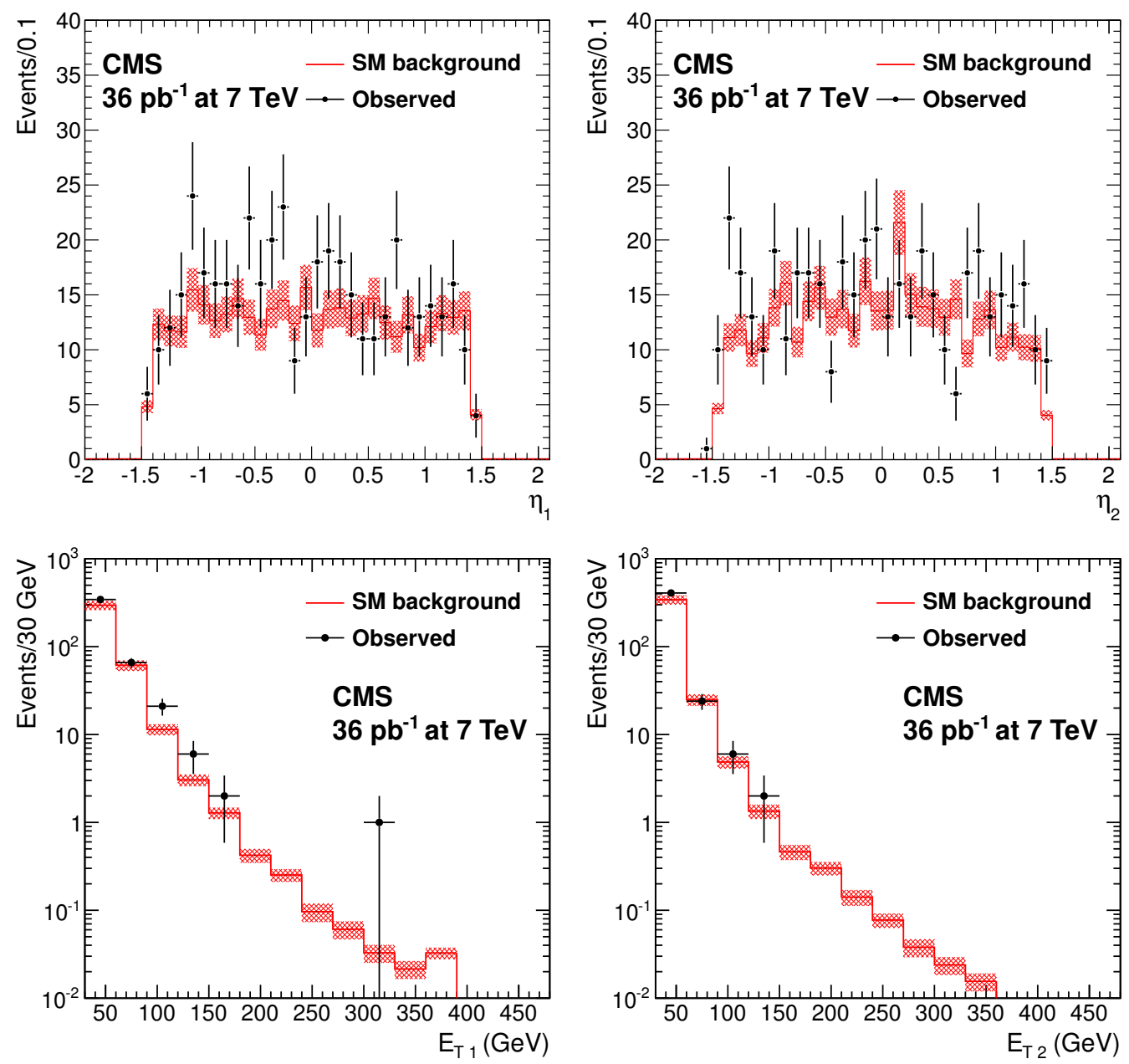

Figure 1. Distributions in $\eta$ and $E_{T}$ for the leading (variable subscript is "1") and sub-leading (variable subscript is "2") photons. Points with error bars represent observed data; the solid histogram corresponds to the expected background from control regions. Shading corresponds to the systematic uncertainty on the background expectation.

and apply a conservative $20 \%$ systematic uncertainty, which is the dominant uncertainty on the background estimation.

The diphoton background is computed with the SHERPA MC program and then rescaled by an NLO $K$ factor of 1.3 [21, 22]. This $K$ factor is alternatively derived with DIPHOx [23], wherein we observe that the $K$ factor decreases slowly as a function of diphoton invariant mass and stabilizes in the range of interest. We therefore use a $K$ factor of $1.3 \pm 0.3$ for the diphoton background to cover its variation as a function of $M_{\gamma \gamma}$ throughout the control, intermediate, and signal regions $\left(M_{\gamma \gamma}>60 \mathrm{GeV}\right)$.

We observe (expect) $440(374 \pm 51)$ events with $M_{\gamma \gamma}>60 \mathrm{GeV}$ and zero $(0.30 \pm 0.07)$ with $M_{\gamma \gamma}>500 \mathrm{GeV}$. Figure 1 shows the pseudorapidity and transverse energy distributions for the photons with the largest and second-largest transverse energy in an event, along with the background predictions. Figure 2 displays the invariant mass distribution 


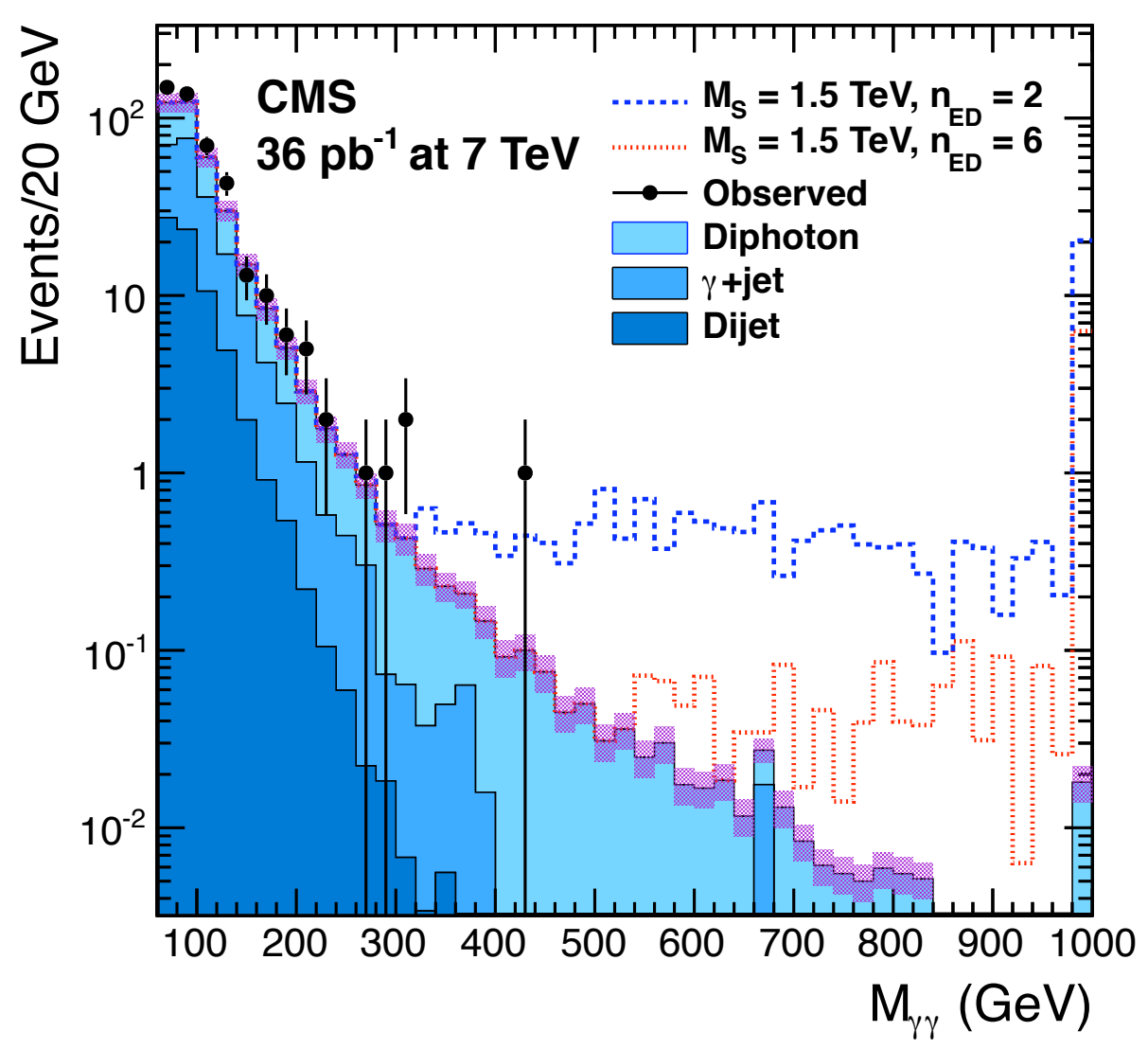

Figure 2. Observed data (points with error bars) and background expectations (filled solid histograms) as a function of the diphoton invariant mass. Photons are required to be isolated, with $E_{\mathrm{T}}>30 \mathrm{GeV}$ and $|\eta|<1.44$. Also shown with dashed lines are the signal distributions for two sets of model parameters in the HLZ convention. Shaded bands around the background estimation correspond to systematic uncertainties. The last bin is an overflow, including the sum of all contributions for $M_{\gamma \gamma}>1.0 \mathrm{TeV}$.

for each of the backgrounds as well as the observed data, with the optimized $\eta$ requirement. Beyond $M_{\gamma \gamma}>200 \mathrm{GeV}$, we see 12 events, consistent with $9.0 \pm 1.5$ expected background events.

Table 1 presents the number of observed data events and expected number of background events in the control, intermediate, and signal regions, respectively. This table corresponds directly to the plot in figure 2. In the control region, we find that the data are consistent with the background expectation within the systematic uncertainty. The systematic uncertainty on the the total background takes into account the correlations between dijet and $\gamma+$ jet backgrounds arising because both depend on the same misidentification rate. The relative combined uncertainty on the backgrounds in the signal region is $23 \%$, due nearly entirely to the diphoton NLO $K$-factor uncertainty. 


\begin{tabular}{|cccc|}
\hline Process & $60<M_{\gamma \gamma}<200 \mathrm{GeV}$ & $200<M_{\gamma \gamma}<500 \mathrm{GeV}$ & $500 \mathrm{GeV}<M_{\gamma \gamma}$ \\
\hline Dijets & $70 \pm 28$ & $0.5 \pm 0.2$ & $0.0009 \pm 0.0004$ \\
$\gamma+$ Jets & $145 \pm 7$ & $2.3 \pm 0.3$ & $0.016 \pm 0.003$ \\
Diphotons & $150 \pm 35$ & $6.2 \pm 1.4$ & $0.29 \pm 0.07$ \\
Total Backgrounds & $365 \pm 49$ & $9.0 \pm 1.5$ & $0.30 \pm 0.07$ \\
\hline Observed & 428 & 12 & 0 \\
\hline
\end{tabular}

Table 1. Data yields and background expectations for reconstructed diphoton invariant mass ranges. Full systematic uncertainties have been included.

\begin{tabular}{|ccc|}
\hline & Central Value & Relative Uncertainty \\
\hline Luminosity & $36 \mathrm{pb}^{-1}$ & $4.0 \%$ \\
Background diphoton $K$ factor & 0.30 Events & $23 \%$ \\
Signal Efficiency & $77.1 \%$ & $5.2 \%$ \\
Signal diphoton $K$ factor & 1.3 & $7.7 \%$ \\
\hline
\end{tabular}

Table 2. Summary of systematic uncertainties in the signal region.

\section{Results}

We perform a counting experiment in the signal region $\left(M_{\gamma \gamma}>500 \mathrm{GeV}\right)$ and set $95 \% \mathrm{CL}$ upper limits on the quantity

$$
S \equiv\left(\sigma_{\text {total }}-\sigma_{\mathrm{SM}}\right) \times \beta \times \mathcal{A},
$$

where $\sigma_{\text {total }}$ represents the total diphoton production cross section (including both signal, $\mathrm{SM}$, and interference effects), and $\sigma_{\mathrm{SM}}$ represents the SM diphoton production cross section. We indicate the signal branching fraction to diphotons by $\beta$ and the signal acceptance by $\mathcal{A}$. We utilize a standard Bayesian approach [3, 24] with a flat prior chosen for the signal cross section and log-normal priors for the nuisance parameters (integrated luminosity, signal efficiency, and background). The likelihood is constructed from the Poisson probability to observe $N$ events, given $S$, the signal efficiency of $(77.1 \pm 4.0) \%$, the expected number of background events $(0.30 \pm 0.07)$, and the integrated luminosity $\mathcal{L}=(36 \pm 1) \mathrm{pb}^{-1}[12]$. Table 2 summarizes the systematic uncertainties used as inputs to the limit calculation.

The observed (expected) 95\% CL upper limit on $S$ is 0.11 (0.13) pb. We translate the limit on $S$ into a limit on the parameters of the ADD model using the following technique. Because the effects of virtual-graviton exchange interfere with the SM diphoton production, generally, we expect the total diphoton cross section to have the following form:

$$
\sigma_{\text {total }}=\sigma_{\mathrm{SM}}+\eta_{G} \sigma_{\mathrm{int}}+\eta_{G}^{2} \sigma_{\mathrm{ED}}
$$

where $\sigma_{\text {int }}$ and $\sigma_{\mathrm{ED}}$ refer to the contributions to the total cross section from interference and direct ED effects, respectively. Here, the dimensionful $\eta_{G}$ parameter specifies the strength 

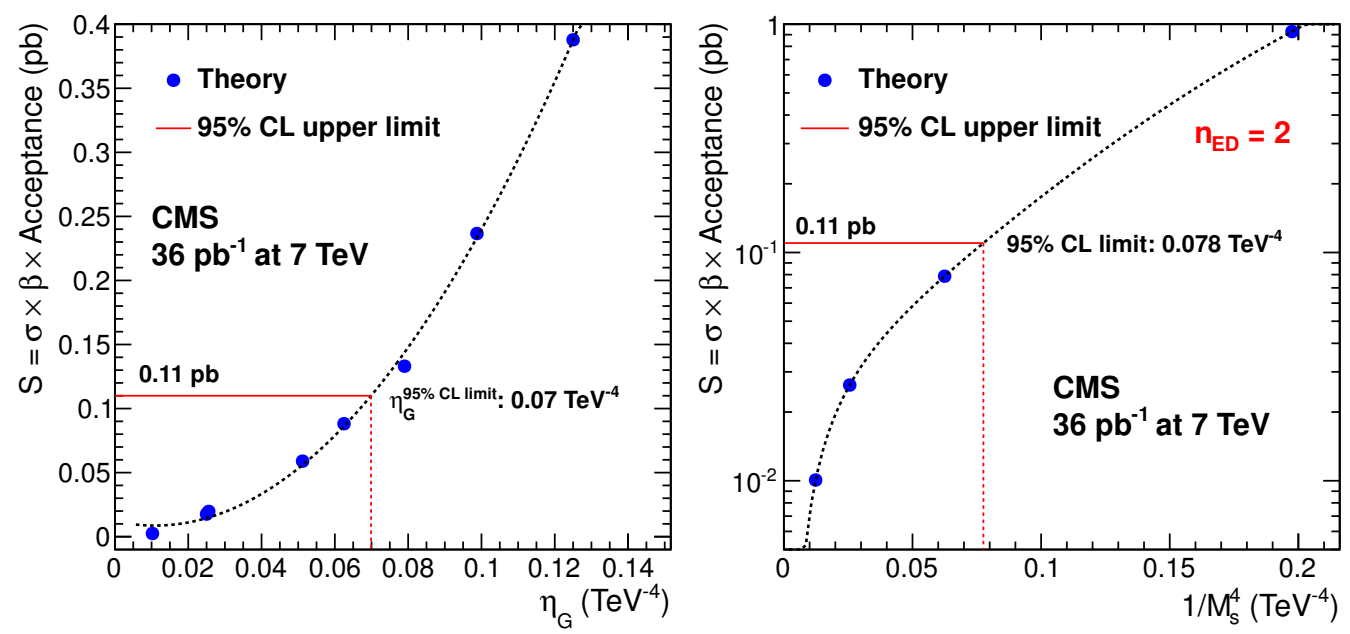

Figure 3. Parameterization of $S$ as a function of the strength of the ED effect, $\eta_{G}$ for all cases except HLZ $n_{\mathrm{ED}}=2$ (left) and as a function of $1 / M_{\mathrm{S}}^{4}$ for the $n_{\mathrm{ED}}=2$ case (right). The parameterization shown is a fit according to eq. (5.2). The solid line is the 95\% CL exclusion limits on $S$, and is matched to the corresponding limits on $\eta_{G}$ and $1 / M_{\mathrm{S}}^{4}$.

\begin{tabular}{|c|c|cc|cccccc|}
\hline & GRW & \multicolumn{2}{|c|}{ Hewett } & \multicolumn{7}{|c|}{ HLZ } \\
& & Pos. Neg. & $n_{\mathrm{ED}}=2$ & $n_{\mathrm{ED}}=3$ & $n_{\mathrm{ED}}=4$ & $n_{\mathrm{ED}}=5$ & $n_{\mathrm{ED}}=6$ & $n_{\mathrm{ED}}=7$ \\
Full & 1.94 & 1.74 & 1.71 & 1.89 & 2.31 & 1.94 & 1.76 & 1.63 & 1.55 \\
Trunc. & 1.84 & 1.60 & 1.50 & 1.80 & 2.23 & 1.84 & 1.63 & 1.46 & 1.31 \\
\hline
\end{tabular}

Table 3. 95\% CL limits on $M_{\mathrm{S}}(\mathrm{TeV})$, as a function of the convention and number of ED. A comparison of the limits with a truncation of the production cross section above $\sqrt{\hat{s}}>M_{\mathrm{S}}$ is also shown. The two limits for the Hewett convention correspond to positive and negative interference effects.

of the ED effects and is related to $M_{\mathrm{S}}$ through eqs. (1.1)-(1.3). Consequently, we parameterize $S$ as a function of the parameter $\eta_{G}$. For the HLZ $n_{\mathrm{ED}}=2$ case, $\eta_{G}$ depends on the signal spectrum due to an explicit $\hat{s}$ dependence in eq. (1.2). Therefore, in this case we parameterize $S$ as a function of $1 / M_{\mathrm{S}}^{4}$ and translate the limit on this parameter into a limit on $M_{\mathrm{S}}$.

The observed 95\% CL upper limit on $S$ together with the parameterization of $S$ as a function of $\eta_{G}$ and $1 / M_{\mathrm{S}}^{4}$ are shown in the left pane of figure 3 . The intersection of the limit on $S$ with the curves determines the upper 95\% CL upper limits on the parameters $\eta_{G}$ and $1 / M_{\mathrm{S}}^{4}$. As seen from these plots, the upper limits are equal to $0.070 \mathrm{TeV}^{-4}$ for $\eta_{G}$ and $0.078 \mathrm{TeV}^{-4}$ for $1 / M_{\mathrm{S}}^{4}$ (for $n_{\mathrm{ED}}=2$ ). We further translate these limits, by means of eq. (1.2), into lower bounds on the fundamental Planck scale for various numbers of extra dimensions, $n_{\mathrm{ED}}$, as shown in table 3 . This is calculated trivially for $n_{\mathrm{ED}}=2$ and for $n_{\mathrm{ED}}>2$ by using eq. (1.2). The limits in the GRW convention are identical to the HLZ limits for $n_{\mathrm{ED}}=4$; the limit for the Hewett convention with constructive interference is $1.74 \mathrm{TeV}$ and is close to the HLZ limit for $n_{\mathrm{ED}}=5$.

We note that the LO signal cross section calculations become non-perturbative when the value of $\hat{s}$ in the $2 \rightarrow 2$ process exceeds $M_{\mathrm{S}}^{2}$. This effect is not taken into account in 
the SHERPA cross section calculations used in this analysis, or in previous studies of this process at the Tevatron [10], where the effect is not expected to be important due to the lower collider energy. Because the energy of the LHC is significantly higher than the limits on $M_{\mathrm{S}}$ we are able to set in this analysis, we take this effect into account by conservatively assuming that the signal cross section is zero for $\sqrt{\hat{s}}>M_{\mathrm{S}}$. Under these assumptions, the limits on $M_{\mathrm{S}}$ decrease by $5 \%$ for $n_{\mathrm{ED}}=2(1.80 \mathrm{TeV})$ and $15 \%$ for $n_{\mathrm{ED}}=7(1.31 \mathrm{TeV})$. A summary of the limits under the assumption of a truncated production cross section is also shown in table 3 .

In addition to setting limits on a specific model of large extra dimensions, we can also set a model-independent limit on any new physics model which results in central, high- $E_{\mathrm{T}}$ diphotons, either resonant or non-resonant (e.g., Kaluza-Klein gravitons in the RandallSundrum model [25]). The measured upper limit of $0.11 \mathrm{pb}$ applies also to the cross section times branching fraction times acceptance of diphoton pairs with $M_{\gamma \gamma}>500 \mathrm{GeV}$ and the following kinematic requirements on each of the two photons: $E_{\mathrm{T}}>30 \mathrm{GeV}$ and $|\eta|<1.44$.

\section{Conclusions}

In conclusion, we have performed a search for large extra dimensions $[1,2]$ in the diphoton final state with a data sample collected in pp collisions at $\sqrt{s}=7 \mathrm{TeV}$ corresponding to an integrated luminosity of $36 \mathrm{pb}^{-1}$. We optimize the signal selection to reach maximum sensitivity in a counting experiment in a one-sided mass window by selecting events with centrally produced photons and large diphoton invariant mass. Given the absence of an excess over the SM direct diphoton background, we set lower limits on the cutoff scale $M_{\mathrm{S}}$ in the range 1.6-2.3 TeV. While this analysis was being finalized, a phenomenological interpretation of the dijet angular distribution results from the CMS and ATLAS experiments appeared [26] and suggested even stronger limits on $M_{\mathrm{S}}$. However, a dedicated experimental analysis and interpretation of the dijet data in the models with large extra dimensions has yet to be conducted. The results presented in this paper extend the current limits reached at the Tevatron $[9,10]$ in all but the $n_{\mathrm{ED}}=2$ case.

\section{Acknowledgments}

We wish to congratulate our colleagues in the CERN accelerator departments for the excellent performance of the LHC machine. We thank the technical and administrative staff at CERN and other CMS institutes, and acknowledge support from:FMSR (Austria); FNRS and FWO (Belgium); CNPq, CAPES, FAPERJ, and FAPESP (Brazil); MES (Bulgaria); CERN; CAS, MoST, and NSFC (China); COLCIENCIAS (Colombia); MSES (Croatia); RPF (Cyprus); Academy of Sciences and NICPB (Estonia); Academy of Finland, ME, and HIP (Finland); CEA and CNRS/IN2P3 (France); BMBF, DFG, and HGF (Germany); GSRT (Greece); OTKA and NKTH (Hungary); DAE and DST (India); IPM (Iran); SFI (Ireland); INFN (Italy); NRF and WCU (Korea); LAS (Lithuania); CINVESTAV, CONACYT, SEP, and UASLP-FAI (Mexico); PAEC (Pakistan); SCSR (Poland); FCT (Portugal); JINR (Armenia, Belarus, Georgia, Ukraine, Uzbekistan); MST and MAE 
(Russia); MSTD (Serbia); MICINN and CPAN (Spain); Swiss Funding Agencies (Switzerland); NSC (Taipei); TUBITAK and TAEK (Turkey); STFC (United Kingdom); DOE and NSF (U.S.A.).

\section{The CMS collaboration author list}

\section{Yerevan Physics Institute, Yerevan, Armenia}

S. Chatrchyan, V. Khachatryan, A.M. Sirunyan, A. Tumasyan

\section{Institut für Hochenergiephysik der OeAW, Wien, Austria}

W. Adam, T. Bergauer, M. Dragicevic, J. Erö, C. Fabjan, M. Friedl, R. Frühwirth, V.M. Ghete, J. Hammer ${ }^{1}$, S. Hänsel, M. Hoch, N. Hörmann, J. Hrubec, M. Jeitler, G. Kasieczka, W. Kiesenhofer, M. Krammer, D. Liko, I. Mikulec, M. Pernicka, H. Rohringer, R. Schöfbeck, J. Strauss, F. Teischinger, P. Wagner, W. Waltenberger, G. Walzel, E. Widl, C.-E. Wulz

National Centre for Particle and High Energy Physics, Minsk, Belarus V. Mossolov, N. Shumeiko, J. Suarez Gonzalez

Universiteit Antwerpen, Antwerpen, Belgium

L. Benucci, E.A. De Wolf, X. Janssen, T. Maes, L. Mucibello, S. Ochesanu, B. Roland, R. Rougny, M. Selvaggi, H. Van Haevermaet, P. Van Mechelen, N. Van Remortel

Vrije Universiteit Brussel, Brussel, Belgium

F. Blekman, S. Blyweert, J. D'Hondt, O. Devroede, R. Gonzalez Suarez, A. Kalogeropoulos, J. Maes, M. Maes, W. Van Doninck, P. Van Mulders, G.P. Van Onsem, I. Villella

Université Libre de Bruxelles, Bruxelles, Belgium

O. Charaf, B. Clerbaux, G. De Lentdecker, V. Dero, A.P.R. Gay, G.H. Hammad, T. Hreus, P.E. Marage, L. Thomas, C. Vander Velde, P. Vanlaer

\section{Ghent University, Ghent, Belgium}

V. Adler, A. Cimmino, S. Costantini, M. Grunewald, B. Klein, J. Lellouch, A. Marinov, J. Mccartin, D. Ryckbosch, F. Thyssen, M. Tytgat, L. Vanelderen, P. Verwilligen, S. Walsh, N. Zaganidis

\section{Université Catholique de Louvain, Louvain-la-Neuve, Belgium}

S. Basegmez, G. Bruno, J. Caudron, L. Ceard, E. Cortina Gil, J. De Favereau De Jeneret, C. Delaere ${ }^{1}$, D. Favart, A. Giammanco, G. Grégoire, J. Hollar, V. Lemaitre, J. Liao, O. Militaru, S. Ovyn, D. Pagano, A. Pin, K. Piotrzkowski, N. Schul

Université de Mons, Mons, Belgium

N. Beliy, T. Caebergs, E. Daubie

Centro Brasileiro de Pesquisas Fisicas, Rio de Janeiro, Brazil

G.A. Alves, D. De Jesus Damiao, M.E. Pol, M.H.G. Souza

Universidade do Estado do Rio de Janeiro, Rio de Janeiro, Brazil

W. Carvalho, E.M. Da Costa, C. De Oliveira Martins, S. Fonseca De Souza, L. Mundim, H. Nogima, V. Oguri, W.L. Prado Da Silva, A. Santoro, S.M. Silva Do Amaral, A. Sznajder, F. Torres Da Silva De Araujo 
Instituto de Fisica Teorica, Universidade Estadual Paulista, Sao Paulo, Brazil F.A. Dias, T.R. Fernandez Perez Tomei, E. M. Gregores ${ }^{2}$, C. Lagana, F. Marinho, P.G. Mercadante ${ }^{2}$, S.F. Novaes, Sandra S. Padula

Institute for Nuclear Research and Nuclear Energy, Sofia, Bulgaria

N. Darmenov ${ }^{1}$, L. Dimitrov, V. Genchev ${ }^{1}$, P. Iaydjiev $^{1}$, S. Piperov, M. Rodozov, S. Stoykova, G. Sultanov, V. Tcholakov, R. Trayanov, I. Vankov

University of Sofia, Sofia, Bulgaria

A. Dimitrov, R. Hadjiiska, A. Karadzhinova, V. Kozhuharov, L. Litov, M. Mateev,

B. Pavlov, P. Petkov

Institute of High Energy Physics, Beijing, China

J.G. Bian, G.M. Chen, H.S. Chen, C.H. Jiang, D. Liang, S. Liang, X. Meng, J. Tao,

J. Wang, J. Wang, X. Wang, Z. Wang, H. Xiao, M. Xu, J. Zang, Z. Zhang

State Key Lab. of Nucl. Phys. and Tech., Peking University, Beijing, China

Y. Ban, S. Guo, Y. Guo, W. Li, Y. Mao, S.J. Qian, H. Teng, L. Zhang, B. Zhu, W. Zou

Universidad de Los Andes, Bogota, Colombia

A. Cabrera, B. Gomez Moreno, A.A. Ocampo Rios, A.F. Osorio Oliveros, J.C. Sanabria

Technical University of Split, Split, Croatia

N. Godinovic, D. Lelas, K. Lelas, R. Plestina ${ }^{3}$, D. Polic, I. Puljak

University of Split, Split, Croatia

Z. Antunovic, M. Dzelalija

Institute Rudjer Boskovic, Zagreb, Croatia

V. Brigljevic, S. Duric, K. Kadija, S. Morovic

University of Cyprus, Nicosia, Cyprus

A. Attikis, M. Galanti, J. Mousa, C. Nicolaou, F. Ptochos, P.A. Razis

Charles University, Prague, Czech Republic

M. Finger, M. Finger Jr.

Academy of Scientific Research and Technology of the Arab Republic of Egypt, Egyptian Network of High Energy Physics, Cairo, Egypt

Y. Assran ${ }^{4}$, A. Awad, S. Khalii ${ }^{5}$

National Institute of Chemical Physics and Biophysics, Tallinn, Estonia

A. Hektor, M. Kadastik, M. Müntel, M. Raidal, L. Rebane

Department of Physics, University of Helsinki, Helsinki, Finland

V. Azzolini, P. Eerola, G. Fedi

Helsinki Institute of Physics, Helsinki, Finland

S. Czellar, J. Härkönen, A. Heikkinen, V. Karimäki, R. Kinnunen, M.J. Kortelainen,

T. Lampén, K. Lassila-Perini, S. Lehti, T. Lindén, P. Luukka, T. Mäenpää, E. Tuominen,

J. Tuominiemi, E. Tuovinen, D. Ungaro, L. Wendland

Lappeenranta University of Technology, Lappeenranta, Finland

K. Banzuzi, A. Korpela, T. Tuuva 
Laboratoire d'Annecy-le-Vieux de Physique des Particules, IN2P3-CNRS, Annecy-le-Vieux, France

D. Sillou

DSM/IRFU, CEA/Saclay, Gif-sur-Yvette, France

M. Besancon, S. Choudhury, M. Dejardin, D. Denegri, B. Fabbro, J.L. Faure, F. Ferri,

S. Ganjour, F.X. Gentit, A. Givernaud, P. Gras, G. Hamel de Monchenault, P. Jarry,

E. Locci, J. Malcles, M. Marionneau, L. Millischer, J. Rander, A. Rosowsky, I. Shreyber,

M. Titov, P. Verrecchia

Laboratoire Leprince-Ringuet, Ecole Polytechnique, IN2P3-CNRS, Palaiseau, France

S. Baffioni, F. Beaudette, L. Benhabib, L. Bianchini, M. Bluj ${ }^{6}$, C. Broutin, P. Busson, C. Charlot, T. Dahms, L. Dobrzynski, S. Elgammal, R. Granier de Cassagnac, M. Haguenauer, P. Miné, C. Mironov, C. Ochando, P. Paganini, D. Sabes, R. Salerno, Y. Sirois, C. Thiebaux, B. Wyslouch ${ }^{7}$, A. Zabi

Institut Pluridisciplinaire Hubert Curien, Université de Strasbourg, Université de Haute Alsace Mulhouse, CNRS/IN2P3, Strasbourg, France

J.-L. Agram ${ }^{8}$, J. Andrea, D. Bloch, D. Bodin, J.-M. Brom, M. Cardaci, E.C. Chabert, C. Collard, E. Conte $^{8}$, F. Drouhin ${ }^{8}$, C. Ferro, J.-C. Fontaine ${ }^{8}$, D. Gelé, U. Goerlach, S. Greder, P. Juillot, M. Karim ${ }^{8}$, A.-C. Le Bihan, Y. Mikami, P. Van Hove

Centre de Calcul de l'Institut National de Physique Nucleaire et de Physique des Particules (IN2P3), Villeurbanne, France

F. Fassi, D. Mercier

Université de Lyon, Université Claude Bernard Lyon 1, CNRS-IN2P3, Institut de Physique Nucléaire de Lyon, Villeurbanne, France

C. Baty, S. Beauceron, N. Beaupere, M. Bedjidian, O. Bondu, G. Boudoul, D. Boumediene,

H. Brun, N. Chanon, R. Chierici, D. Contardo, P. Depasse, H. El Mamouni, J. Fay,

S. Gascon, B. Ille, T. Kurca, T. Le Grand, M. Lethuillier, L. Mirabito, S. Perries, V. Sordini, S. Tosi, Y. Tschudi, P. Verdier

Institute of High Energy Physics and Informatization, Tbilisi State University, Tbilisi, Georgia

D. Lomidze

RWTH Aachen University, I. Physikalisches Institut, Aachen, Germany

G. Anagnostou, M. Edelhoff, L. Feld, N. Heracleous, O. Hindrichs, R. Jussen, K. Klein, J. Merz, N. Mohr, A. Ostapchuk, A. Perieanu, F. Raupach, J. Sammet, S. Schael, D. Sprenger, H. Weber, M. Weber, B. Wittmer

RWTH Aachen University, III. Physikalisches Institut A, Aachen, Germany M. Ata, W. Bender, E. Dietz-Laursonn, M. Erdmann, J. Frangenheim, T. Hebbeker, A. Hinzmann, K. Hoepfner, T. Klimkovich, D. Klingebiel, P. Kreuzer, D. Lanske ${ }^{\dagger}$, C. Magass, M. Merschmeyer, A. Meyer, P. Papacz, H. Pieta, H. Reithler, S.A. Schmitz, L. Sonnenschein, J. Steggemann, D. Teyssier, M. Tonutti 
RWTH Aachen University, III. Physikalisches Institut B, Aachen, Germany M. Bontenackels, M. Davids, M. Duda, G. Flügge, H. Geenen, M. Giffels, W. Haj Ahmad, D. Heydhausen, T. Kress, Y. Kuessel, A. Linn, A. Nowack, L. Perchalla, O. Pooth, J. Rennefeld, P. Sauerland, A. Stahl, M. Thomas, D. Tornier, M.H. Zoeller

Deutsches Elektronen-Synchrotron, Hamburg, Germany

M. Aldaya Martin, W. Behrenhoff, U. Behrens, M. Bergholz ${ }^{9}$, K. Borras, A. Cakir, A. Campbell, E. Castro, D. Dammann, G. Eckerlin, D. Eckstein, A. Flossdorf, G. Flucke, A. Geiser, J. Hauk, H. Jung ${ }^{1}$, M. Kasemann, I. Katkov ${ }^{10}$, P. Katsas, C. Kleinwort, H. Kluge, A. Knutsson, M. Krämer, D. Krücker, E. Kuznetsova, W. Lange, W. Lohmann ${ }^{9}$, R. Mankel, M. Marienfeld, I.-A. Melzer-Pellmann, A.B. Meyer, J. Mnich, A. Mussgiller, J. Olzem, D. Pitzl, A. Raspereza, A. Raval, M. Rosin, R. Schmidt ${ }^{9}$, T. Schoerner-Sadenius, N. Sen, A. Spiridonov, M. Stein, J. Tomaszewska, R. Walsh, C. Wissing

University of Hamburg, Hamburg, Germany

C. Autermann, V. Blobel, S. Bobrovskyi, J. Draeger, H. Enderle, U. Gebbert, K. Kaschube, G. Kaussen, R. Klanner, J. Lange, B. Mura, S. Naumann-Emme, F. Nowak, N. Pietsch, C. Sander, H. Schettler, P. Schleper, M. Schröder, T. Schum, J. Schwandt, H. Stadie, G. Steinbrück, J. Thomsen

Institut für Experimentelle Kernphysik, Karlsruhe, Germany

C. Barth, J. Bauer, V. Buege, T. Chwalek, W. De Boer, A. Dierlamm, G. Dirkes, M. Feindt, J. Gruschke, C. Hackstein, F. Hartmann, M. Heinrich, H. Held, K.H. Hoffmann, S. Honc, J.R. Komaragiri, T. Kuhr, D. Martschei, S. Mueller, Th. Müller, M. Niegel, O. Oberst, A. Oehler, J. Ott, T. Peiffer, D. Piparo, G. Quast, K. Rabbertz, F. Ratnikov, N. Ratnikova, M. Renz, C. Saout, A. Scheurer, P. Schieferdecker, F.-P. Schilling, M. Schmanau, G. Schott, H.J. Simonis, F.M. Stober, D. Troendle, J. Wagner-Kuhr, T. Weiler, M. Zeise, V. Zhukov ${ }^{10}$, E.B. Ziebarth

Institute of Nuclear Physics "Demokritos", Aghia Paraskevi, Greece

G. Daskalakis, T. Geralis, K. Karafasoulis, S. Kesisoglou, A. Kyriakis, D. Loukas, I. Manolakos, A. Markou, C. Markou, C. Mavrommatis, E. Ntomari, E. Petrakou

University of Athens, Athens, Greece

L. Gouskos, T.J. Mertzimekis, A. Panagiotou, E. Stiliaris

University of Ioánnina, Ioánnina, Greece

I. Evangelou, C. Foudas, P. Kokkas, N. Manthos, I. Papadopoulos, V. Patras, F.A. Triantis

KFKI Research Institute for Particle and Nuclear Physics, Budapest, Hungary

A. Aranyi, G. Bencze, L. Boldizsar, C. Hajdu ${ }^{1}$, P. Hidas, D. Horvath ${ }^{11}$, A. Kapusi, K. Krajczar ${ }^{12}$, B. Radics, F. Sikler ${ }^{1}$, G.I. Veres ${ }^{12}$, G. Vesztergombi ${ }^{12}$

Institute of Nuclear Research ATOMKI, Debrecen, Hungary

N. Beni, J. Molnar, J. Palinkas, Z. Szillasi, V. Veszpremi

University of Debrecen, Debrecen, Hungary

P. Raics, Z.L. Trocsanyi, B. Ujvari 
Panjab University, Chandigarh, India

S. Bansal, S.B. Beri, V. Bhatnagar, N. Dhingra, R. Gupta, M. Jindal, M. Kaur, J.M. Kohli, M.Z. Mehta, N. Nishu, L.K. Saini, A. Sharma, A.P. Singh, J.B. Singh, S.P. Singh

University of Delhi, Delhi, India

S. Ahuja, S. Bhattacharya, B.C. Choudhary, P. Gupta, S. Jain, S. Jain, A. Kumar, K. Ranjan, R.K. Shivpuri

Bhabha Atomic Research Centre, Mumbai, India

R.K. Choudhury, D. Dutta, S. Kailas, V. Kumar, A.K. Mohanty ${ }^{1}$, L.M. Pant, P. Shukla

Tata Institute of Fundamental Research - EHEP, Mumbai, India

T. Aziz, M. Guchait ${ }^{13}$, A. Gurtu, M. Maity ${ }^{14}$, D. Majumder, G. Majumder, K. Mazumdar,

G.B. Mohanty, A. Saha, K. Sudhakar, N. Wickramage

Tata Institute of Fundamental Research - HECR, Mumbai, India

S. Banerjee, S. Dugad, N.K. Mondal

Institute for Research and Fundamental Sciences (IPM), Tehran, Iran

H. Arfaei, H. Bakhshiansohi ${ }^{15}$, S.M. Etesami, A. Fahim ${ }^{15}$, M. Hashemi, A. Jafari ${ }^{15}$, M. Khakzad, A. Mohammadi ${ }^{16}$, M. Mohammadi Najafabadi, S. Paktinat Mehdiabadi, B. Safarzadeh, M. Zeinali ${ }^{17}$

INFN Sezione di Bari ${ }^{a}$, Università di Bari ${ }^{b}$, Politecnico di Bari ${ }^{c}$, Bari, Italy M. Abbrescia ${ }^{a, b}$, L. Barbone ${ }^{a, b}$, C. Calabria ${ }^{a, b}$, A. Colaleo ${ }^{a}$, D. Creanza ${ }^{a, c}$, N. De Filippis $^{a, c, 1}$, M. De Palma ${ }^{a, b}$, L. Fiore $^{a}$, G. Iaselli ${ }^{a, c}$, L. Lusito $^{a, b}$, G. Maggi $^{a, c}$, M. Maggi $^{a}$, N. Manna ${ }^{a, b}$, B. Marangellia ${ }^{a, b}$, S. $\mathrm{My}^{a, c}$, S. Nuzzo ${ }^{a, b}$, N. Pacifico ${ }^{a, b}$, G.A. Pierro ${ }^{a}$, A. Pompili ${ }^{a, b}$, G. Pugliese ${ }^{a, c}$, F. Romano ${ }^{a, c}$, G. Roselli ${ }^{a, b}$, G. Selvaggi ${ }^{a}, b$, L. Silvestris ${ }^{a}$, R. Trentadue ${ }^{a}$, S. Tupputi ${ }^{a}, b$, G. Zito ${ }^{a}$

INFN Sezione di Bologna ${ }^{a}$, Università di Bologna ${ }^{b}$, Bologna, Italy

G. Abbiendi ${ }^{a}$, A.C. Benvenuti ${ }^{a}$, D. Bonacorsi ${ }^{a}$, S. Braibant-Giacomelli $^{a}, b$, L. Brigliadori ${ }^{a}$, P. Capiluppi ${ }^{a, b}$, A. Castro ${ }^{a, b}$, F.R. Cavallo ${ }^{a}$, M. Cuffiani ${ }^{a, b}$, G.M. Dallavalle ${ }^{a}$, F. Fabbri ${ }^{a}$, A. Fanfani ${ }^{a}, b$, D. Fasanella ${ }^{a}$, P. Giacomelli ${ }^{a}$, M. Giunta ${ }^{a}$, S. Marcellini ${ }^{a}$, G. Masetti, M. Meneghelli ${ }^{a, b}$, A. Montanari ${ }^{a}$, F.L. Navarria ${ }^{a, b}$, F. Odorici ${ }^{a}$, A. Perrotta $^{a}$,

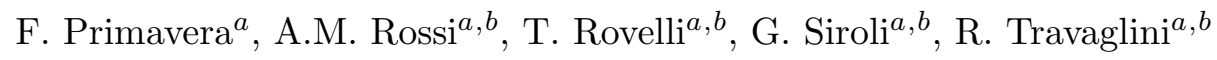

INFN Sezione di Catania ${ }^{a}$, Università di Catania ${ }^{b}$, Catania, Italy

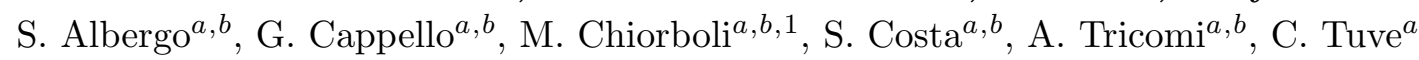

INFN Sezione di Firenze ${ }^{a}$, Università di Firenze ${ }^{b}$, Firenze, Italy

G. Barbagli ${ }^{a}$, V. Ciulli ${ }^{a}, b$, C. Civinini ${ }^{a}$, R. D’Alessandro ${ }^{a, b}$, E. Focardi ${ }^{a, b}$, S. Frosali ${ }^{a}, b$,

E. Gallo ${ }^{a}$, S. Gonzi ${ }^{a, b}$, P. Lenzi ${ }^{a, b}$, M. Meschini ${ }^{a}$, S. Paoletti ${ }^{a}$, G. Sguazzoni ${ }^{a}$, A. Tropiano ${ }^{a, 1}$

INFN Laboratori Nazionali di Frascati, Frascati, Italy

L. Benussi, S. Bianco, S. Colafranceschi ${ }^{18}$, F. Fabbri, D. Piccolo

INFN Sezione di Genova, Genova, Italy

P. Fabbricatore, R. Musenich 


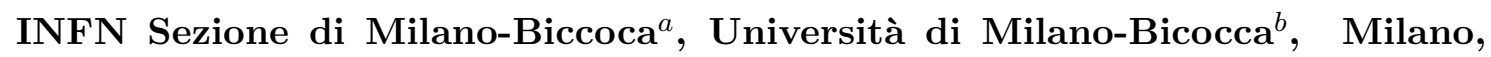
Italy

A. Benaglia ${ }^{a, b}$, F. De Guio ${ }^{a, b, 1}$, L. Di Matteo ${ }^{a, b}$, A. Ghezzi ${ }^{a, b}$, M. Malberti ${ }^{a}, b$, S. Malvezzi ${ }^{a}$, A. Martelli ${ }^{a, b}$, A. Massironi ${ }^{a, b}$, D. Menasce ${ }^{a}$, L. Moroni ${ }^{a}$, M. Paganoni ${ }^{a}, b$, D. Pedrini ${ }^{a}$, S. Ragazzi ${ }^{a, b}$, N. Redaelli ${ }^{a}$, S. Sala ${ }^{a}$, T. Tabarelli de Fatis ${ }^{a, b}$, V. Tancini ${ }^{a, b}$

INFN Sezione di Napoli ${ }^{a}$, Università di Napoli "Federico II" ${ }^{b}$, Napoli, Italy

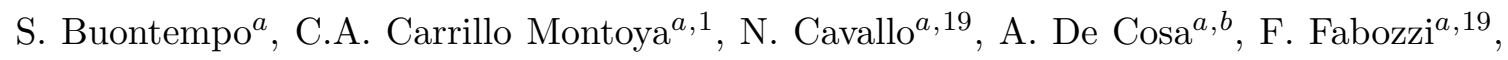

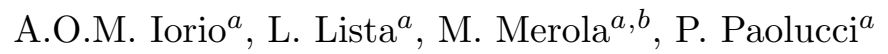

INFN Sezione di Padova ${ }^{a}$, Università di Padova ${ }^{b}$, Università di Trento (Trento) ${ }^{c}$, Padova, Italy

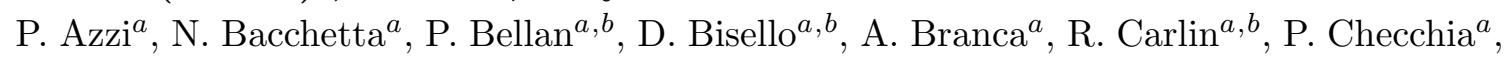

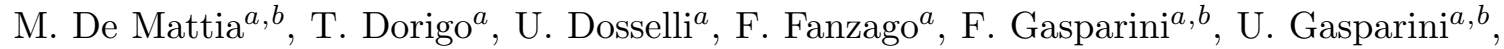
S. Lacaprara ${ }^{a, 20}$, I. Lazzizzera ${ }^{a, c}$, M. Margoni ${ }^{a, b}$, M. Mazzucato ${ }^{a}$, A.T. Meneguzzo ${ }^{a, b}$, M. Nespolo ${ }^{a, 1}$, L. Perrozzi ${ }^{a, 1}$, N. Pozzobon ${ }^{a, b}$, P. Ronchese $^{a, b}$, F. Simonetto ${ }^{a, b}$, E. Torassa $^{a}$, M. Tosi ${ }^{a, b}$, S. Vanini ${ }^{a, b}$, P. Zotto ${ }^{a, b}$, G. Zumerle ${ }^{a, b}$

INFN Sezione di Pavia ${ }^{a}$, Università di Pavia ${ }^{b}$, Pavia, Italy

P. Baesso ${ }^{a, b}$, U. Berzano ${ }^{a}$, S.P. Ratti ${ }^{a, b}$, C. Riccardi ${ }^{a, b}$, P. Torre ${ }^{a, b}$, P. Vitulo ${ }^{a, b}$, C. Viviani ${ }^{a, b}$

INFN Sezione di Perugia ${ }^{a}$, Università di Perugia ${ }^{b}$, Perugia, Italy

M. Biasini ${ }^{a, b}$, G.M. Bilei ${ }^{a}$, B. Caponeri ${ }^{a, b}$, L. Fanò ${ }^{a, b}$, P. Lariccia ${ }^{a, b}$, A. Lucaroni ${ }^{a, b, 1}$, G. Mantovani ${ }^{a, b}$, M. Menichelli ${ }^{a}$, A. Nappi $^{a, b}$, F. Romeo $^{a, b}$, A. Santocchia $^{a, b}$, S. Taroni ${ }^{a, b, 1}$, M. Valdata ${ }^{a, b}$

INFN Sezione di Pisa ${ }^{a}$, Università di Pisa ${ }^{b}$, Scuola Normale Superiore di Pisa ${ }^{c}$, Pisa, Italy

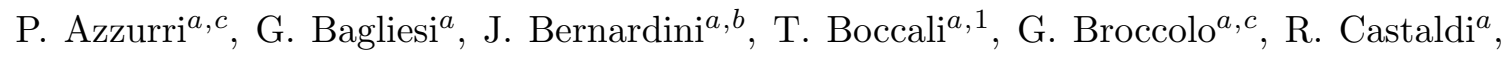

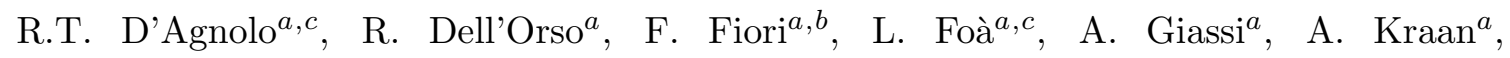

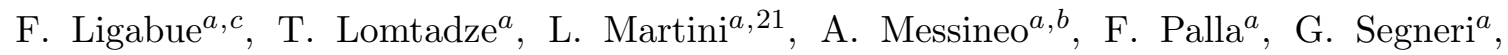

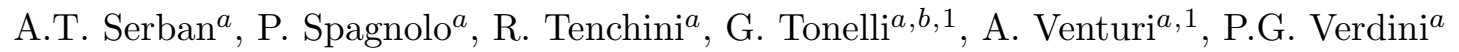

INFN Sezione di Roma ${ }^{a}$, Università di Roma "La Sapienza" ${ }^{b}$, Roma, Italy L. Barone $e^{a, b}$, F. Cavallari ${ }^{a}$, D. Del Re ${ }^{a, b}$, E. Di Marco ${ }^{a, b}$, M. Diemoz $^{a}$, D. Franci ${ }^{a, b}$, M. Grassi ${ }^{a, 1}$, E. Longo $^{a, b}$, S. Nourbakhsh ${ }^{a}$, G. Organtini ${ }^{a, b}$, F. Pandolfi ${ }^{a, b, 1}$, R. Paramatti $^{a}$, S. Rahatlou ${ }^{a, b}$

INFN Sezione di Torino ${ }^{a}$, Università di Torino ${ }^{b}$, Università del Piemonte Orientale (Novara) ${ }^{c}$, Torino, Italy

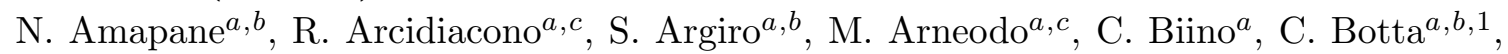

N. Cartiglia ${ }^{a}$, R. Castello ${ }^{a, b}$, M. Costa ${ }^{a, b}$, N. Demaria ${ }^{a}$, A. Graziano ${ }^{a, b, 1}$, C. Mariotti ${ }^{a}$, M. Marone ${ }^{a, b}$, S. Maselli ${ }^{a}$, E. Migliore ${ }^{a, b}$, G. Mila ${ }^{a, b}$, V. Monaco ${ }^{a, b}$, M. Musich ${ }^{a, b}$, M.M. Obertino ${ }^{a, c}$, N. Pastrone ${ }^{a}$, M. Pelliccioni ${ }^{a, b}$, A. Romero $^{a, b}$, M. Ruspa $^{a, c}$, R. Sacchi $^{a, b}$, V. Sola ${ }^{a, b}$, A. Solano ${ }^{a, b}$, A. Staiano ${ }^{a}$, D. Trocino ${ }^{a, b}$, A. Vilela Pereira ${ }^{a, b}$ 
INFN Sezione di Trieste ${ }^{a}$, Università di Trieste ${ }^{b}$, Trieste, Italy

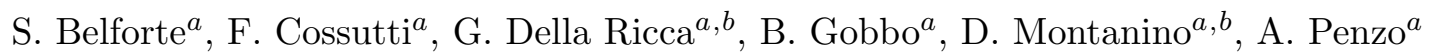

Kangwon National University, Chunchon, Korea

S.G. Heo, S.K. Nam

Kyungpook National University, Daegu, Korea

S. Chang, J. Chung, D.H. Kim, G.N. Kim, J.E. Kim, D.J. Kong, H. Park, S.R. Ro, D. Son, D.C. Son, T. Son

Chonnam National University, Institute for Universe and Elementary Particles, Kwangju, Korea

Zero Kim, J.Y. Kim, S. Song

Korea University, Seoul, Korea

S. Choi, B. Hong, M.S. Jeong, M. Jo, H. Kim, J.H. Kim, T.J. Kim, K.S. Lee, D.H. Moon, S.K. Park, H.B. Rhee, E. Seo, S. Shin, K.S. Sim

University of Seoul, Seoul, Korea

M. Choi, S. Kang, H. Kim, C. Park, I.C. Park, S. Park, G. Ryu

Sungkyunkwan University, Suwon, Korea

Y. Choi, Y.K. Choi, J. Goh, M.S. Kim, E. Kwon, J. Lee, S. Lee, H. Seo, I. Yu

Vilnius University, Vilnius, Lithuania

M.J. Bilinskas, I. Grigelionis, M. Janulis, D. Martisiute, P. Petrov, T. Sabonis

Centro de Investigacion y de Estudios Avanzados del IPN, Mexico City, Mexico

H. Castilla-Valdez, E. De La Cruz-Burelo, R. Lopez-Fernandez, A. Sánchez-Hernández, L.M. Villasenor-Cendejas

Universidad Iberoamericana, Mexico City, Mexico

S. Carrillo Moreno, F. Vazquez Valencia

Benemerita Universidad Autonoma de Puebla, Puebla, Mexico

H.A. Salazar Ibarguen

Universidad Autónoma de San Luis Potosí, San Luis Potosí, Mexico

E. Casimiro Linares, A. Morelos Pineda, M.A. Reyes-Santos

University of Auckland, Auckland, New Zealand

D. Krofcheck, J. Tam

University of Canterbury, Christchurch, New Zealand

P.H. Butler, R. Doesburg, H. Silverwood

National Centre for Physics, Quaid-I-Azam University, Islamabad, Pakistan

M. Ahmad, I. Ahmed, M.I. Asghar, H.R. Hoorani, W.A. Khan, T. Khurshid, S. Qazi

Institute of Experimental Physics, Faculty of Physics, University of Warsaw, Warsaw, Poland

M. Cwiok, W. Dominik, K. Doroba, A. Kalinowski, M. Konecki, J. Krolikowski 
Soltan Institute for Nuclear Studies, Warsaw, Poland

T. Frueboes, R. Gokieli, M. Górski, M. Kazana, K. Nawrocki, K. Romanowska-Rybinska,

M. Szleper, G. Wrochna, P. Zalewski

Laboratório de Instrumentação e Física Experimental de Partículas, Lisboa, Portugal

N. Almeida, P. Bargassa, A. David, P. Faccioli, P.G. Ferreira Parracho, M. Gallinaro, P. Musella, A. Nayak, J. Seixas, J. Varela

Joint Institute for Nuclear Research, Dubna, Russia

S. Afanasiev, I. Belotelov, P. Bunin, I. Golutvin, A. Kamenev, V. Karjavin, G. Kozlov,

A. Lanev, P. Moisenz, V. Palichik, V. Perelygin, S. Shmatov, V. Smirnov, A. Volodko,

A. Zarubin

Petersburg Nuclear Physics Institute, Gatchina (St Petersburg), Russia

V. Golovtsov, Y. Ivanov, V. Kim, P. Levchenko, V. Murzin, V. Oreshkin, I. Smirnov, V. Sulimov, L. Uvarov, S. Vavilov, A. Vorobyev, A. Vorobyev

Institute for Nuclear Research, Moscow, Russia

Yu. Andreev, A. Dermenev, S. Gninenko, N. Golubev, M. Kirsanov, N. Krasnikov, V. Matveev, A. Pashenkov, A. Toropin, S. Troitsky

Institute for Theoretical and Experimental Physics, Moscow, Russia

V. Epshteyn, V. Gavrilov, V. Kaftanov ${ }^{\dagger}$, M. Kossov ${ }^{1}$, A. Krokhotin, N. Lychkovskaya, V. Popov, G. Safronov, S. Semenov, V. Stolin, E. Vlasov, A. Zhokin

Moscow State University, Moscow, Russia

E. Boos, M. Dubinin ${ }^{22}$, L. Dudko, A. Ershov, A. Gribushin, O. Kodolova, I. Lokhtin, A. Markina, S. Obraztsov, M. Perfilov, S. Petrushanko, L. Sarycheva, V. Savrin, A. Snigirev

P.N. Lebedev Physical Institute, Moscow, Russia

V. Andreev, M. Azarkin, I. Dremin, M. Kirakosyan, A. Leonidov, S.V. Rusakov, A. Vinogradov

State Research Center of Russian Federation, Institute for High Energy Physics, Protvino, Russia

I. Azhgirey, S. Bitioukov, V. Grishin ${ }^{1}$, V. Kachanov, D. Konstantinov, A. Korablev, V. Krychkine, V. Petrov, R. Ryutin, S. Slabospitsky, A. Sobol, L. Tourtchanovitch, S. Troshin, N. Tyurin, A. Uzunian, A. Volkov

University of Belgrade, Faculty of Physics and Vinca Institute of Nuclear Sciences, Belgrade, Serbia

P. Adzic ${ }^{23}$, M. Djordjevic, D. Krpic ${ }^{23}$, J. Milosevic

Centro de Investigaciones Energéticas Medioambientales y Tecnológicas (CIEMAT), Madrid, Spain

M. Aguilar-Benitez, J. Alcaraz Maestre, P. Arce, C. Battilana, E. Calvo, M. Cepeda, M. Cerrada, M. Chamizo Llatas, N. Colino, B. De La Cruz, A. Delgado Peris, C. Diez Pardos, D. Domínguez Vázquez, C. Fernandez Bedoya, J.P. Fernández Ramos, A. Ferrando, 
J. Flix, M.C. Fouz, P. Garcia-Abia, O. Gonzalez Lopez, S. Goy Lopez, J.M. Hernandez, M.I. Josa, G. Merino, J. Puerta Pelayo, I. Redondo, L. Romero, J. Santaolalla, M.S. Soares, C. Willmott

Universidad Autónoma de Madrid, Madrid, Spain

C. Albajar, G. Codispoti, J.F. de Trocóniz

Universidad de Oviedo, Oviedo, Spain

J. Cuevas, J. Fernandez Menendez, S. Folgueras, I. Gonzalez Caballero, L. Lloret Iglesias, J.M. Vizan Garcia

Instituto de Física de Cantabria (IFCA), CSIC-Universidad de Cantabria, Santander, Spain

J.A. Brochero Cifuentes, I.J. Cabrillo, A. Calderon, S.H. Chuang, J. Duarte Campderros, M. Felcini ${ }^{24}$, M. Fernandez, G. Gomez, J. Gonzalez Sanchez, C. Jorda, P. Lobelle Pardo, A. Lopez Virto, J. Marco, R. Marco, C. Martinez Rivero, F. Matorras, F.J. Munoz Sanchez, J. Piedra Gomez ${ }^{25}$, T. Rodrigo, A.Y. Rodríguez-Marrero, A. Ruiz-Jimeno, L. Scodellaro, M. Sobron Sanudo, I. Vila, R. Vilar Cortabitarte

CERN, European Organization for Nuclear Research, Geneva, Switzerland

D. Abbaneo, E. Auffray, G. Auzinger, P. Baillon, A.H. Ball, D. Barney, A.J. Bell ${ }^{26}$, D. Benedetti, C. Bernet ${ }^{3}$, W. Bialas, P. Bloch, A. Bocci, S. Bolognesi, M. Bona, H. Breuker, G. Brona, K. Bunkowski, T. Camporesi, G. Cerminara, J.A. Coarasa Perez, B. Curé, D. D'Enterria, A. De Roeck, S. Di Guida, A. Elliott-Peisert, B. Frisch, W. Funk, A. Gaddi, S. Gennai, G. Georgiou, H. Gerwig, D. Gigi, K. Gill, D. Giordano, F. Glege, R. Gomez-Reino Garrido, M. Gouzevitch, P. Govoni, S. Gowdy, L. Guiducci, M. Hansen, C. Hartl, J. Harvey, J. Hegeman, B. Hegner, H.F. Hoffmann, A. Honma, V. Innocente, P. Janot, K. Kaadze, E. Karavakis, P. Lecoq, C. Lourenço, T. Mäki, L. Malgeri, M. Mannelli, L. Masetti, A. Maurisset, F. Meijers, S. Mersi, E. Meschi, R. Moser, M.U. Mozer, M. Mulders, E. Nesvold ${ }^{1}$, M. Nguyen, T. Orimoto, L. Orsini, E. Perez, A. Petrilli, A. Pfeiffer, M. Pierini, M. Pimiä, G. Polese, A. Racz, J. Rodrigues Antunes, G. Rolandi ${ }^{27}$, T. Rommerskirchen, C. Rovelli, M. Rovere, H. Sakulin, C. Schäfer, C. Schwick, I. Segoni, A. Sharma, P. Siegrist, M. Simon, P. Sphicas ${ }^{28}$, M. Spiropulu ${ }^{22}$, M. Stoye, P. Tropea, A. Tsirou, P. Vichoudis, M. Voutilainen, W.D. Zeuner

\section{Paul Scherrer Institut, Villigen, Switzerland}

W. Bertl, K. Deiters, W. Erdmann, K. Gabathuler, R. Horisberger, Q. Ingram, H.C. Kaestli, S. König, D. Kotlinski, U. Langenegger, F. Meier, D. Renker, T. Rohe, J. Sibille ${ }^{29}$, A. Starodumov ${ }^{30}$

Institute for Particle Physics, ETH Zurich, Zurich, Switzerland

P. Bortignon, L. Caminada ${ }^{31}$, Z. Chen, S. Cittolin, G. Dissertori, M. Dittmar, J. Eugster, K. Freudenreich, C. Grab, A. Hervé, W. Hintz, P. Lecomte, W. Lustermann, C. Marchica ${ }^{31}$, P. Martinez Ruiz del Arbol, P. Meridiani, P. Milenovic ${ }^{32}$, F. Moortgat, C. Nägeli ${ }^{31}$, P. Nef, F. Nessi-Tedaldi, L. Pape, F. Pauss, T. Punz, A. Rizzi, F.J. Ronga, M. Rossini, L. Sala, A.K. Sanchez, M.-C. Sawley, B. Stieger, L. Tauscher ${ }^{\dagger}$, A. Thea, K. Theofilatos, D. Treille, C. Urscheler, R. Wallny, M. Weber, L. Wehrli, J. Weng 


\section{Universität Zürich, Zurich, Switzerland}

E. Aguiló, C. Amsler, V. Chiochia, S. De Visscher, C. Favaro, M. Ivova Rikova, B. Millan Mejias, P. Otiougova, C. Regenfus, P. Robmann, A. Schmidt, H. Snoek

\section{National Central University, Chung-Li, Taiwan}

Y.H. Chang, K.H. Chen, C.M. Kuo, S.W. Li, W. Lin, Z.K. Liu, Y.J. Lu, D. Mekterovic, R. Volpe, J.H. Wu, S.S. Yu

\section{National Taiwan University (NTU), Taipei, Taiwan}

P. Bartalini, P. Chang, Y.H. Chang, Y.W. Chang, Y. Chao, K.F. Chen, W.-S. Hou, Y. Hsiung, K.Y. Kao, Y.J. Lei, R.-S. Lu, J.G. Shiu, Y.M. Tzeng, M. Wang

\section{Cukurova University, Adana, Turkey}

A. Adiguzel, M.N. Bakirci ${ }^{33}$, S. Cerci ${ }^{34}$, C. Dozen, I. Dumanoglu, E. Eskut, S. Girgis, G. Gokbulut, Y. Guler, E. Gurpinar, I. Hos, E.E. Kangal, T. Karaman, A. Kayis Topaksu, A. Nart, G. Onengut, K. Ozdemir, S. Ozturk, A. Polatoz, K. Sogut ${ }^{35}$, D. Sunar Cerci ${ }^{34}$, B. Tali, H. Topakli ${ }^{33}$, D. Uzun, L.N. Vergili, M. Vergili, C. Zorbilmez

Middle East Technical University, Physics Department, Ankara, Turkey I.V. Akin, T. Aliev, S. Bilmis, M. Deniz, H. Gamsizkan, A.M. Guler, K. Ocalan, A. Ozpineci, M. Serin, R. Sever, U.E. Surat, E. Yildirim, M. Zeyrek

Bogazici University, Istanbul, Turkey

M. Deliomeroglu, D. Demir ${ }^{36}$, E. Gülmez, B. Isildak, M. Kaya ${ }^{37}$, O. Kaya ${ }^{37}$, S. Ozkorucuklu ${ }^{38}$, N. Sonmez ${ }^{39}$

National Scientific Center, Kharkov Institute of Physics and Technology, Kharkov, Ukraine

L. Levchuk

University of Bristol, Bristol, U.K.

F. Bostock, J.J. Brooke, T.L. Cheng, E. Clement, D. Cussans, R. Frazier, J. Goldstein, M. Grimes, M. Hansen, D. Hartley, G.P. Heath, H.F. Heath, J. Jackson, L. Kreczko, S. Metson, D.M. Newbold ${ }^{40}$, K. Nirunpong, A. Poll, S. Senkin, V.J. Smith, S. Ward

Rutherford Appleton Laboratory, Didcot, U.K.

L. Basso ${ }^{41}$, K.W. Bell, A. Belyaev ${ }^{41}$, C. Brew, R.M. Brown, B. Camanzi, D.J.A. Cockerill, J.A. Coughlan, K. Harder, S. Harper, B.W. Kennedy, E. Olaiya, D. Petyt, B.C. RadburnSmith, C.H. Shepherd-Themistocleous, I.R. Tomalin, W.J. Womersley, S.D. Worm

\section{Imperial College, London, U.K.}

R. Bainbridge, G. Ball, J. Ballin, R. Beuselinck, O. Buchmuller, D. Colling, N. Cripps, M. Cutajar, G. Davies, M. Della Negra, W. Ferguson, J. Fulcher, D. Futyan, A. Gilbert, A. Guneratne Bryer, G. Hall, Z. Hatherell, J. Hays, G. Iles, M. Jarvis, G. Karapostoli, L. Lyons, B.C. MacEvoy, A.-M. Magnan, J. Marrouche, B. Mathias, R. Nandi, J. Nash, A. Nikitenko ${ }^{30}$, A. Papageorgiou, M. Pesaresi, K. Petridis, M. Pioppi ${ }^{42}$, D.M. Raymond, S. Rogerson, N. Rompotis, A. Rose, M.J. Ryan, C. Seez, P. Sharp, A. Sparrow, A. Tapper, S. Tourneur, M. Vazquez Acosta, T. Virdee, S. Wakefield, N. Wardle, D. Wardrope, T. Whyntie 
Brunel University, Uxbridge, U.K.

M. Barrett, M. Chadwick, J.E. Cole, P.R. Hobson, A. Khan, P. Kyberd, D. Leslie,

W. Martin, I.D. Reid, L. Teodorescu

Baylor University, Waco, U.S.A.

K. Hatakeyama

Boston University, Boston, U.S.A.

T. Bose, E. Carrera Jarrin, C. Fantasia, A. Heister, J. St. John, P. Lawson, D. Lazic, J. Rohlf, D. Sperka, L. Sulak

Brown University, Providence, U.S.A.

A. Avetisyan, S. Bhattacharya, J.P. Chou, D. Cutts, A. Ferapontov, U. Heintz, S. Jabeen,

G. Kukartsev, G. Landsberg, M. Narain, D. Nguyen, M. Segala, T. Speer, K.V. Tsang

University of California, Davis, Davis, U.S.A.

R. Breedon, M. Calderon De La Barca Sanchez, S. Chauhan, M. Chertok, J. Conway, P.T. Cox, J. Dolen, R. Erbacher, E. Friis, W. Ko, A. Kopecky, R. Lander, H. Liu, S. Maruyama, T. Miceli, M. Nikolic, D. Pellett, J. Robles, S. Salur, T. Schwarz, M. Searle, J. Smith, M. Squires, M. Tripathi, R. Vasquez Sierra, C. Veelken

University of California, Los Angeles, Los Angeles, U.S.A.

V. Andreev, K. Arisaka, D. Cline, R. Cousins, A. Deisher, J. Duris, S. Erhan, C. Farrell, J. Hauser, M. Ignatenko, C. Jarvis, C. Plager, G. Rakness, P. Schlein ${ }^{\dagger}$, J. Tucker, V. Valuev

University of California, Riverside, Riverside, U.S.A.

J. Babb, A. Chandra, R. Clare, J. Ellison, J.W. Gary, F. Giordano, G. Hanson, G.Y. Jeng, S.C. Kao, F. Liu, H. Liu, O.R. Long, A. Luthra, H. Nguyen, B.C. Shen ${ }^{\dagger}$, R. Stringer, J. Sturdy, S. Sumowidagdo, R. Wilken, S. Wimpenny

University of California, San Diego, La Jolla, U.S.A.

W. Andrews, J.G. Branson, G.B. Cerati, E. Dusinberre, D. Evans, F. Golf, A. Holzner, R. Kelley, M. Lebourgeois, J. Letts, B. Mangano, S. Padhi, C. Palmer, G. Petrucciani, H. Pi, M. Pieri, R. Ranieri, M. Sani, V. Sharma, S. Simon, Y. Tu, A. Vartak, S. Wasserbaech ${ }^{43}$, F. Würthwein, A. Yagil, J. Yoo

University of California, Santa Barbara, Santa Barbara, U.S.A.

D. Barge, R. Bellan, C. Campagnari, M. D’Alfonso, T. Danielson, K. Flowers, P. Geffert, J. Incandela, C. Justus, P. Kalavase, S.A. Koay, D. Kovalskyi, V. Krutelyov, S. Lowette, N. Mccoll, V. Pavlunin, F. Rebassoo, J. Ribnik, J. Richman, R. Rossin, D. Stuart, W. To, J.R. Vlimant

California Institute of Technology, Pasadena, U.S.A.

A. Apresyan, A. Bornheim, J. Bunn, Y. Chen, M. Gataullin, Y. Ma, A. Mott, H.B. Newman, C. Rogan, K. Shin, V. Timciuc, P. Traczyk, J. Veverka, R. Wilkinson, Y. Yang, R.Y. Zhu

Carnegie Mellon University, Pittsburgh, U.S.A.

B. Akgun, R. Carroll, T. Ferguson, Y. Iiyama, D.W. Jang, S.Y. Jun, Y.F. Liu, M. Paulini, J. Russ, H. Vogel, I. Vorobiev 
University of Colorado at Boulder, Boulder, U.S.A.

J.P. Cumalat, M.E. Dinardo, B.R. Drell, C.J. Edelmaier, W.T. Ford, A. Gaz, B. Heyburn, E. Luiggi Lopez, U. Nauenberg, J.G. Smith, K. Stenson, K.A. Ulmer, S.R. Wagner, S.L. Zang

Cornell University, Ithaca, U.S.A.

L. Agostino, J. Alexander, D. Cassel, A. Chatterjee, S. Das, N. Eggert, L.K. Gibbons, B. Heltsley, W. Hopkins, A. Khukhunaishvili, B. Kreis, G. Nicolas Kaufman, J.R. Patterson, D. Puigh, A. Ryd, X. Shi, W. Sun, W.D. Teo, J. Thom, J. Thompson, J. Vaughan, Y. Weng, L. Winstrom, P. Wittich

Fairfield University, Fairfield, U.S.A.

A. Biselli, G. Cirino, D. Winn

Fermi National Accelerator Laboratory, Batavia, U.S.A.

S. Abdullin, M. Albrow, J. Anderson, G. Apollinari, M. Atac, J.A. Bakken, S. Banerjee, L.A.T. Bauerdick, A. Beretvas, J. Berryhill, P.C. Bhat, I. Bloch, F. Borcherding, K. Burkett, J.N. Butler, V. Chetluru, H.W.K. Cheung, F. Chlebana, S. Cihangir, W. Cooper, D.P. Eartly, V.D. Elvira, S. Esen, I. Fisk, J. Freeman, Y. Gao, E. Gottschalk, D. Green, K. Gunthoti, O. Gutsche, J. Hanlon, R.M. Harris, J. Hirschauer, B. Hooberman, H. Jensen, M. Johnson, U. Joshi, R. Khatiwada, B. Klima, K. Kousouris, S. Kunori, S. Kwan, C. Leonidopoulos, P. Limon, D. Lincoln, R. Lipton, J. Lykken, K. Maeshima, J.M. Marraffino, D. Mason, P. McBride, T. Miao, K. Mishra, S. Mrenna, Y. Musienko ${ }^{44}$, C. Newman-Holmes, V. O'Dell, R. Pordes, O. Prokofyev, N. Saoulidou, E. Sexton-Kennedy, S. Sharma, W.J. Spalding, L. Spiegel, P. Tan, L. Taylor, S. Tkaczyk, L. Uplegger, E.W. Vaandering, R. Vidal, J. Whitmore, W. Wu, F. Yang, F. Yumiceva, J.C. Yun

University of Florida, Gainesville, U.S.A.

D. Acosta, P. Avery, D. Bourilkov, M. Chen, M. De Gruttola, G.P. Di Giovanni, D. Dobur, A. Drozdetskiy, R.D. Field, M. Fisher, Y. Fu, I.K. Furic, J. Gartner, B. Kim, J. Konigsberg, A. Korytov, A. Kropivnitskaya, T. Kypreos, K. Matchev, G. Mitselmakher, L. Muniz, Y. Pakhotin, C. Prescott, R. Remington, M. Schmitt, B. Scurlock, P. Sellers, N. Skhirtladze, M. Snowball, D. Wang, J. Yelton, M. Zakaria

Florida International University, Miami, U.S.A.

C. Ceron, V. Gaultney, L. Kramer, L.M. Lebolo, S. Linn, P. Markowitz, G. Martinez, D. Mesa, J.L. Rodriguez

Florida State University, Tallahassee, U.S.A.

T. Adams, A. Askew, D. Bandurin, J. Bochenek, J. Chen, B. Diamond, S.V. Gleyzer, J. Haas, S. Hagopian, V. Hagopian, M. Jenkins, K.F. Johnson, H. Prosper, L. Quertenmont, S. Sekmen, V. Veeraraghavan

Florida Institute of Technology, Melbourne, U.S.A.

M.M. Baarmand, B. Dorney, S. Guragain, M. Hohlmann, H. Kalakhety, R. Ralich, I. Vodopiyanov 
University of Illinois at Chicago (UIC), Chicago, U.S.A.

M.R. Adams, I.M. Anghel, L. Apanasevich, Y. Bai, V.E. Bazterra, R.R. Betts, J. Callner, R. Cavanaugh, C. Dragoiu, L. Gauthier, C.E. Gerber, D.J. Hofman, S. Khalatyan, G.J. Kunde ${ }^{45}$, F. Lacroix, M. Malek, C. O'Brien, C. Silvestre, A. Smoron, D. Strom, N. Varelas

\section{The University of Iowa, Iowa City, U.S.A.}

U. Akgun, E.A. Albayrak, B. Bilki, W. Clarida, F. Duru, C.K. Lae, E. McCliment, J.-P. Merlo, H. Mermerkaya ${ }^{46}$, A. Mestvirishvili, A. Moeller, J. Nachtman, C.R. Newsom, E. Norbeck, J. Olson, Y. Onel, F. Ozok, S. Sen, J. Wetzel, T. Yetkin, K. Yi

Johns Hopkins University, Baltimore, U.S.A.

B.A. Barnett, B. Blumenfeld, A. Bonato, C. Eskew, D. Fehling, G. Giurgiu, A.V. Gritsan, Z.J. Guo, G. Hu, P. Maksimovic, S. Rappoccio, M. Swartz, N.V. Tran, A. Whitbeck

The University of Kansas, Lawrence, U.S.A.

P. Baringer, A. Bean, G. Benelli, O. Grachov, M. Murray, D. Noonan, S. Sanders, J.S. Wood, V. Zhukova

Kansas State University, Manhattan, U.S.A.

A.f. Barfuss, T. Bolton, I. Chakaberia, A. Ivanov, S. Khalil, M. Makouski, Y. Maravin, S. Shrestha, I. Svintradze, Z. Wan

Lawrence Livermore National Laboratory, Livermore, U.S.A.

J. Gronberg, D. Lange, D. Wright

University of Maryland, College Park, U.S.A.

A. Baden, M. Boutemeur, S.C. Eno, D. Ferencek, J.A. Gomez, N.J. Hadley, R.G. Kellogg, M. Kirn, Y. Lu, A.C. Mignerey, K. Rossato, P. Rumerio, F. Santanastasio, A. Skuja, J. Temple, M.B. Tonjes, S.C. Tonwar, E. Twedt

Massachusetts Institute of Technology, Cambridge, U.S.A.

B. Alver, G. Bauer, J. Bendavid, W. Busza, E. Butz, I.A. Cali, M. Chan, V. Dutta, P. Everaerts, G. Gomez Ceballos, M. Goncharov, K.A. Hahn, P. Harris, Y. Kim, M. Klute, Y.-J. Lee, W. Li, C. Loizides, P.D. Luckey, T. Ma, S. Nahn, C. Paus, D. Ralph, C. Roland, G. Roland, M. Rudolph, G.S.F. Stephans, F. Stöckli, K. Sumorok, K. Sung, E.A. Wenger, S. Xie, M. Yang, Y. Yilmaz, A.S. Yoon, M. Zanetti

University of Minnesota, Minneapolis, U.S.A.

P. Cole, S.I. Cooper, P. Cushman, B. Dahmes, A. De Benedetti, P.R. Dudero, G. Franzoni, J. Haupt, K. Klapoetke, Y. Kubota, J. Mans, V. Rekovic, R. Rusack, M. Sasseville, A. Singovsky

University of Mississippi, University, U.S.A.

L.M. Cremaldi, R. Godang, R. Kroeger, L. Perera, R. Rahmat, D.A. Sanders, D. Summers

University of Nebraska-Lincoln, Lincoln, U.S.A.

K. Bloom, S. Bose, J. Butt, D.R. Claes, A. Dominguez, M. Eads, J. Keller, T. Kelly, I. Kravchenko, J. Lazo-Flores, H. Malbouisson, S. Malik, G.R. Snow 
State University of New York at Buffalo, Buffalo, U.S.A.

U. Baur, A. Godshalk, I. Iashvili, S. Jain, A. Kharchilava, A. Kumar, S.P. Shipkowski,

K. Smith

Northeastern University, Boston, U.S.A.

G. Alverson, E. Barberis, D. Baumgartel, O. Boeriu, M. Chasco, S. Reucroft, J. Swain,

D. Wood, J. Zhang

Northwestern University, Evanston, U.S.A.

A. Anastassov, A. Kubik, N. Odell, R.A. Ofierzynski, B. Pollack, A. Pozdnyakov, M. Schmitt, S. Stoynev, M. Velasco, S. Won

University of Notre Dame, Notre Dame, U.S.A.

L. Antonelli, D. Berry, M. Hildreth, C. Jessop, D.J. Karmgard, J. Kolb, T. Kolberg, K. Lannon, W. Luo, S. Lynch, N. Marinelli, D.M. Morse, T. Pearson, R. Ruchti, J. Slaunwhite, N. Valls, M. Wayne, J. Ziegler

The Ohio State University, Columbus, U.S.A.

B. Bylsma, L.S. Durkin, J. Gu, C. Hill, P. Killewald, K. Kotov, T.Y. Ling, M. Rodenburg,

G. Williams

Princeton University, Princeton, U.S.A.

N. Adam, E. Berry, P. Elmer, D. Gerbaudo, V. Halyo, P. Hebda, A. Hunt, J. Jones, E. Laird, D. Lopes Pegna, D. Marlow, T. Medvedeva, M. Mooney, J. Olsen, P. Piroué, X. Quan, H. Saka, D. Stickland, C. Tully, J.S. Werner, A. Zuranski

University of Puerto Rico, Mayaguez, U.S.A.

J.G. Acosta, X.T. Huang, A. Lopez, H. Mendez, S. Oliveros, J.E. Ramirez Vargas, A. Zatserklyaniy

Purdue University, West Lafayette, U.S.A.

E. Alagoz, V.E. Barnes, G. Bolla, L. Borrello, D. Bortoletto, A. Everett, A.F. Garfinkel, L. Gutay, Z. Hu, M. Jones, O. Koybasi, M. Kress, A.T. Laasanen, N. Leonardo, C. Liu, V. Maroussov, P. Merkel, D.H. Miller, N. Neumeister, I. Shipsey, D. Silvers, A. Svyatkovskiy, H.D. Yoo, J. Zablocki, Y. Zheng

Purdue University Calumet, Hammond, U.S.A.

P. Jindal, N. Parashar

Rice University, Houston, U.S.A.

C. Boulahouache, V. Cuplov, K.M. Ecklund, F.J.M. Geurts, B.P. Padley, R. Redjimi, J. Roberts, J. Zabel

University of Rochester, Rochester, U.S.A.

B. Betchart, A. Bodek, Y.S. Chung, R. Covarelli, P. de Barbaro, R. Demina, Y. Eshaq,

H. Flacher, A. Garcia-Bellido, P. Goldenzweig, Y. Gotra, J. Han, A. Harel, D.C. Miner,

D. Orbaker, G. Petrillo, D. Vishnevskiy, M. Zielinski

The Rockefeller University, New York, U.S.A.

A. Bhatti, R. Ciesielski, L. Demortier, K. Goulianos, G. Lungu, S. Malik, C. Mesropian, M. Yan 
Rutgers, the State University of New Jersey, Piscataway, U.S.A.

O. Atramentov, A. Barker, D. Duggan, Y. Gershtein, R. Gray, E. Halkiadakis, D. Hidas,

D. Hits, A. Lath, S. Panwalkar, R. Patel, A. Richards, K. Rose, S. Schnetzer, S. Somalwar,

R. Stone, S. Thomas

University of Tennessee, Knoxville, U.S.A.

G. Cerizza, M. Hollingsworth, S. Spanier, Z.C. Yang, A. York

Texas A\&M University, College Station, U.S.A.

J. Asaadi, R. Eusebi, J. Gilmore, A. Gurrola, T. Kamon, V. Khotilovich, R. Montalvo, C.N. Nguyen, I. Osipenkov, J. Pivarski, A. Safonov, S. Sengupta, A. Tatarinov, D. Toback, M. Weinberger

Texas Tech University, Lubbock, U.S.A.

N. Akchurin, C. Bardak, J. Damgov, C. Jeong, K. Kovitanggoon, S.W. Lee, Y. Roh, A. Sill, I. Volobouev, R. Wigmans, E. Yazgan

Vanderbilt University, Nashville, U.S.A.

E. Appelt, E. Brownson, D. Engh, C. Florez, W. Gabella, M. Issah, W. Johns, P. Kurt,

C. Maguire, A. Melo, P. Sheldon, B. Snook, S. Tuo, J. Velkovska

University of Virginia, Charlottesville, U.S.A.

M.W. Arenton, M. Balazs, S. Boutle, B. Cox, B. Francis, R. Hirosky, A. Ledovskoy, C. Lin, C. Neu, R. Yohay

Wayne State University, Detroit, U.S.A.

S. Gollapinni, R. Harr, P.E. Karchin, P. Lamichhane, M. Mattson, C. Milstène, A. Sakharov

University of Wisconsin, Madison, U.S.A.

M. Anderson, M. Bachtis, J.N. Bellinger, D. Carlsmith, S. Dasu, J. Efron, K. Flood,

L. Gray, K.S. Grogg, M. Grothe, R. Hall-Wilton, M. Herndon, P. Klabbers, J. Klukas,

A. Lanaro, C. Lazaridis, J. Leonard, R. Loveless, A. Mohapatra, F. Palmonari, D. Reeder,

I. Ross, A. Savin, W.H. Smith, J. Swanson, M. Weinberg

${ }^{\dagger}$ Deceased

${ }^{1}$ Also at CERN, European Organization for Nuclear Research, Geneva, Switzerland

${ }^{2}$ Also at Universidade Federal do ABC, Santo Andre, Brazil

${ }^{3}$ Also at Laboratoire Leprince-Ringuet, Ecole Polytechnique, IN2P3-CNRS, Palaiseau, France

${ }^{4}$ Also at Suez Canal University, Suez, Egypt

${ }^{5}$ Also at British University, Cairo, Egypt

${ }^{6}$ Also at Soltan Institute for Nuclear Studies, Warsaw, Poland

${ }^{7}$ Also at Massachusetts Institute of Technology, Cambridge, U.S.A.

${ }^{8}$ Also at Université de Haute-Alsace, Mulhouse, France

${ }^{9}$ Also at Brandenburg University of Technology, Cottbus, Germany

${ }^{10}$ Also at Moscow State University, Moscow, Russia

${ }^{11}$ Also at Institute of Nuclear Research ATOMKI, Debrecen, Hungary 
${ }^{12}$ Also at Eötvös Loránd University, Budapest, Hungary

${ }^{13}$ Also at Tata Institute of Fundamental Research - HECR, Mumbai, India

${ }^{14}$ Also at University of Visva-Bharati, Santiniketan, India

${ }^{15}$ Also at Sharif University of Technology, Tehran, Iran

${ }^{16}$ Also at Shiraz University, Shiraz, Iran

${ }^{17}$ Also at Isfahan University of Technology, Isfahan, Iran

${ }^{18}$ Also at Facoltà Ingegneria Università di Roma "La Sapienza", Roma, Italy

${ }^{19}$ Also at Università della Basilicata, Potenza, Italy

${ }^{20}$ Also at Laboratori Nazionali di Legnaro dell' INFN, Legnaro, Italy

${ }^{21}$ Also at Università degli studi di Siena, Siena, Italy

${ }^{22}$ Also at California Institute of Technology, Pasadena, U.S.A.

${ }^{23}$ Also at Faculty of Physics of University of Belgrade, Belgrade, Serbia

${ }^{24}$ Also at University of California, Los Angeles, Los Angeles, U.S.A.

${ }^{25}$ Also at University of Florida, Gainesville, U.S.A.

${ }^{26}$ Also at Université de Genève, Geneva, Switzerland

${ }^{27}$ Also at Scuola Normale e Sezione dell' INFN, Pisa, Italy

${ }^{28}$ Also at University of Athens, Athens, Greece

${ }^{29}$ Also at The University of Kansas, Lawrence, U.S.A.

${ }^{30} \mathrm{Also}$ at Institute for Theoretical and Experimental Physics, Moscow, Russia

${ }^{31}$ Also at Paul Scherrer Institut, Villigen, Switzerland

${ }^{32}$ Also at University of Belgrade, Faculty of Physics and Vinca Institute of Nuclear Sciences, Belgrade, Serbia

${ }^{33}$ Also at Gaziosmanpasa University, Tokat, Turkey

${ }^{34}$ Also at Adiyaman University, Adiyaman, Turkey

${ }^{35}$ Also at Mersin University, Mersin, Turkey

${ }^{36}$ Also at Izmir Institute of Technology, Izmir, Turkey

${ }^{37}$ Also at Kafkas University, Kars, Turkey

${ }^{38}$ Also at Suleyman Demirel University, Isparta, Turkey

${ }^{39} \mathrm{Also}$ at Ege University, Izmir, Turkey

${ }^{40} \mathrm{Also}$ at Rutherford Appleton Laboratory, Didcot, U.K.

${ }^{41}$ Also at School of Physics and Astronomy, University of Southampton, Southampton, U.K.

${ }^{42}$ Also at INFN Sezione di Perugia; Università di Perugia, Perugia, Italy

${ }^{43}$ Also at Utah Valley University, Orem, U.S.A.

${ }^{44}$ Also at Institute for Nuclear Research, Moscow, Russia

${ }^{45}$ Also at Los Alamos National Laboratory, Los Alamos, U.S.A.

${ }^{46}$ Also at Erzincan University, Erzincan, Turkey

Open Access. This article is distributed under the terms of the Creative Commons Attribution Noncommercial License which permits any noncommercial use, distribution, and reproduction in any medium, provided the original author(s) and source are credited. 


\section{References}

[1] N. Arkani-Hamed, S. Dimopoulos and G.R. Dvali, The hierarchy problem and new dimensions at a millimeter, Phys. Lett. B 429 (1998) 263 [hep-ph/9803315] [SPIRES].

[2] N. Arkani-Hamed, S. Dimopoulos and G.R. Dvali, Phenomenology, astrophysics and cosmology of theories with sub-millimeter dimensions and TeV scale quantum gravity, Phys. Rev. D 59 (1999) 086004 [hep-ph/9807344] [SPIRES].

[3] Particle Data Group collaboration, K. Nakamura et al., Review of particle physics, J. Phys. G 37 (2010) 075021 [SPIRES].

[4] K.-M. Cheung and G.L. Landsberg, Drell-Yan and diphoton production at hadron colliders and low scale gravity model, Phys. Rev. D 62 (2000) 076003 [hep-ph/9909218] [SPIRES].

[5] G.F. Giudice, R. Rattazzi and J.D. Wells, Quantum gravity and extra dimensions at high-energy colliders, Nucl. Phys. B 544 (1999) 3 [hep-ph/9811291] [SPIRES].

[6] T. Han, J.D. Lykken and R.-J. Zhang, On Kaluza-Klein states from large extra dimensions, Phys. Rev. D 59 (1999) 105006 [hep-ph/9811350] [SPIRES].

[7] J.L. Hewett, Indirect collider signals for extra dimensions, Phys. Rev. Lett. 82 (1999) 4765 [hep-ph/9811356] [SPIRES].

[8] G. Landsberg, Collider searches for extra spatial dimensions and black holes, arXiv:0808.1867 [SPIRES].

[9] D0 collaboration, V.M. Abazov et al., Measurement of dijet angular distributions at $\sqrt{s}=1.96$ TeV and searches for quark compositeness and extra spatial dimensions, Phys. Rev. Lett. 103 (2009) 191803 [arXiv:0906.4819] [SPIRES].

[10] D0 collaboration, V.M. Abazov et al., Search for large extra spatial dimensions in the

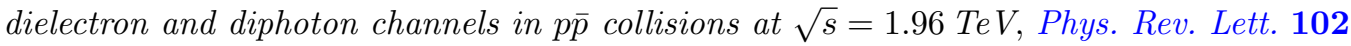
(2009) 051601 [arXiv:0809.2813] [SPIRES].

[11] CMS collaboration, R. Adolphi et al., The CMS experiment at the CERN LHC, 2008 JINST 3 S08004 [SPIRES].

[12] CMS collaboration, Measurement of CMS luminosity, CMS-DP-2011-002, document in preparation, CERN, Geneva Switzerland (2011).

[13] CMS collaboration, V. Khachatryan et al., CMS tracking performance results from early LHC operation, Eur. Phys. J. C 70 (2010) 1165 [arXiv:1007.1988] [SPIRES].

[14] CMS collaboration, Isolated photon reconstruction and identification at $\sqrt{s}=7$ TeV, CMS Physics Analysis Summary, CMS-PAS-EGM-10-006, http://cdsweb.cern.ch/record/1324545, CERN, Geneva Switzerland (2010).

[15] CMS collaboration, V. Khachatryan et al., Measurement of the isolated prompt photon production cross section in pp collisions at $\sqrt{s}=7$ TeV, Phys. Rev. Lett. 106 (2011) 082001 [arXiv:1012.0799] [SPIRES].

[16] CMS collaboration, S. Chatrchyan et al., Time reconstruction and performance of the CMS electromagnetic calorimeter, 2010 JINST 5 T03011 [arXiv:0911.4044] [SPIRES].

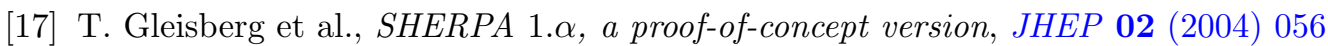
[hep-ph/0311263] [SPIRES]. 
[18] D. Orbaker, Fast simulation of the CMS detector, J. Phys. Conf. Ser. 219 (2010) 032053 [SPIRES].

[19] GEANT4 collaboration, S. Agostinelli et al., GEANT4: a simulation toolkit, Nucl. Instrum. Meth. A 506 (2003) 250 [SPIRES].

[20] CTEQ collaboration, P.M. Nadolsky et al., Implications of CTEQ global analysis for collider observables, Phys. Rev. D 78 (2008) 013004 [arXiv:0802.0007] [SPIRES].

[21] M.C. Kumar, P. Mathews, V. Ravindran and A. Tripathi, Diphoton signals in theories with large extra dimensions to NLO QCD at hadron colliders, Phys. Lett. B 672 (2009) 45 [arXiv: 0811.1670] [SPIRES].

[22] M.C. Kumar, P. Mathews, V. Ravindran and A. Tripathi, Direct photon pair production at the LHC to $O\left(\alpha_{s}\right)$ in TeV scale gravity models, Nucl. Phys. B 818 (2009) 28 [arXiv: 0902.4894] [SPIRES].

[23] T. Binoth, J.P. Guillet, E. Pilon and M. Werlen, A full next-to-leading order study of direct photon pair production in hadronic collisions, Eur. Phys. J. C 16 (2000) 311 [hep-ph/9911340] [SPIRES].

[24] I. Bertram et al., A recipe for the construction of confidence limits, technical report, FERMILAB-TM-2104, Fermilab, Batavia U.S.A. (2000) [SPIRES]

[25] L. Randall and R. Sundrum, A large mass hierarchy from a small extra dimension, Phys. Rev. Lett. 83 (1999) 3370 [hep-ph/9905221] [SPIRES].

[26] R. Franceschini, G.F. Giudice, P.P. Giardino, P. Lodone and A. Strumia, LHC bounds on large extra dimensions, arXiv:1101.4919 [SPIRES]. 